Review

\title{
Antibacterial Activity of Essential Oils and Their Isolated Constituents against Cariogenic Bacteria: A Systematic Review
}

\author{
Irlan Almeida Freires ${ }^{1, \dagger}$, Carina Denny ${ }^{1, \dagger}$, Bruna Benso ${ }^{1, \dagger}$, Severino Matias de Alencar ${ }^{2, \dagger}$ and \\ Pedro Luiz Rosalen $1, \dagger, *$
}

1 Department of Physiological Sciences, Piracicaba Dental School, University of Campinas, Piracicaba, SP 13414-903, Brazil; E-Mails: irlan.almeida@gmail.com (I.A.F.); dennycarina@hotmail.com (C.D.); brunabenso@hotmail.com (B.B.)

2 Department of Agri-food Industry, Food and Nutrition, "Luiz de Queiroz" College of Agriculture, University of São Paulo, Piracicaba, SP 13418-260, Brazil; E-Mail: smalencar@usp.br

$\dagger$ These authors contributed equally to this work.

* Author to whom correspondence should be addressed; E-Mail: rosalen@fop.unicamp.br; Tel.: +55-19-2106-5313; Fax: +55-19-2106-5308.

Academic Editor: Derek J. McPhee

Received: 24 February 2015 / Accepted: 10 April 2015 / Published: 22 April 2015

\begin{abstract}
Dental caries remains the most prevalent and costly oral infectious disease worldwide. Several methods have been employed to prevent this biofilm-dependent disease, including the use of essential oils (EOs). In this systematic review, we discuss the antibacterial activity of EOs and their isolated constituents in view of a potential applicability in novel dental formulations. Seven databases were systematically searched for clinical trials, in situ, in vivo and in vitro studies addressing the topic published up to date. Most of the knowledge in the literature is based on in vitro studies assessing the effects of EOs on caries-related streptococci (mainly Streptococcus mutans) and lactobacilli, and on a limited number of clinical trials. The most promising species with antibacterial potential against cariogenic bacteria are: Achillea ligustica, Baccharis dracunculifolia, Croton cajucara, Cryptomeria japonica, Coriandrum sativum, Eugenia caryophyllata, Lippia sidoides, Ocimum americanum, and Rosmarinus officinalis. In some cases, the major phytochemical compounds determine the biological properties of EOs. Menthol and eugenol were considered outstanding compounds demonstrating an antibacterial potential. Only L. sidoides mouthwash (1\%) has shown clinical antimicrobial effects against oral pathogens thus far. This review suggests avenues for further non-clinical
\end{abstract}


and clinical studies with the most promising EOs and their isolated constituents bioprospected worldwide.

Keywords: natural products; essential oils; monoterpenes; dental caries; Streptococcus mutans; preventive dentistry; clinical trials; isolated compounds

\section{Introduction}

Despite the advances in public policies so far, dental caries remains the most prevalent and costly oral infectious disease worldwide [1,2], representing a global public health problem to be managed by authorities and dental professionals [2,3]. Effective caries-preventive methods have been developed and amended in the last decades. It is well known that the chemical control of plaque is an effective strategy to prevent dental caries development [4]. The main chemical agents currently available are fluoride [5], chlorhexidine [6], triclosan, cetylpyridinium chloride and natural products [4,7].

In this context, natural products (plant extracts, essential oils and isolated compounds, and marine products) have been proposed as novel therapeutic agents against dental caries [8], in order to minimize the adverse effects of synthetics [9] (e.g., altered taste, mucosal desquamation and tooth staining) as well as to provide effective and safer alternatives for dental caries management. Examples of these natural products include propolis, black and green tea, cacao bean husk, oat hulls, cranberry, and shells of crustaceans, among several others [8].

Essential oils (EOs) have aroused attention among the naturally-occurring bioactive agents with promising antimicrobial activity $[10,11]$. EOs are a mixture of volatile constituents produced by aromatic plants as secondary metabolites, as a protective mechanism against predators, microorganisms or weather adversities [12,13]. Among the 100,000 known secondary metabolites, EOs account for over 3000 , of which about 300 have commercial interest and are used by the food, cosmetic and pharmaceutical industries [10]. The diverse chemical structures of EOs encompass two groups with distinct biosynthetic origins [14]: terpenes (monoterpenes and sesquiterpenes) and terpenoids (isoprenoids), and another group of aliphatic and aromatic compounds (e.g., aldehydes, phenols, among others), all characterized by low molecular weight [12]. Monoterpenes are the major compounds found in EOs [12] and have been found to show potent antibacterial activity against caries-related microorganisms $[11,15]$.

Despite the research progress so far, there have been few studies with EOs approaching their potential application in the field of dentistry. Usually, a few substances from this phytochemical class have been used in anti-plaque and anti-gingivitis mouthwash formulations [16-18], hence there is a need for further exploration of EOs with potential use as adjunctive anti-caries chemotherapy.

In this systematic review, we discuss the anti-caries activity of EOs in view of their potential applicability in novel dental formulations. Moreover, the compilation of a vast database from the literature may suggest avenues for further laboratorial and clinical studies with the most promising EOs and their isolated constituents bioprospected worldwide. 


\section{Results}

According to a previously set strategy, literature searches resulted in 1405 articles, of which 25 met the inclusion criteria and were included in the final review after thorough analysis (Figure 1). A total of 22 in vitro studies and three clinical trials addressing the anti-caries properties of EOs and their isolated compounds were selected and will be further discussed herein.
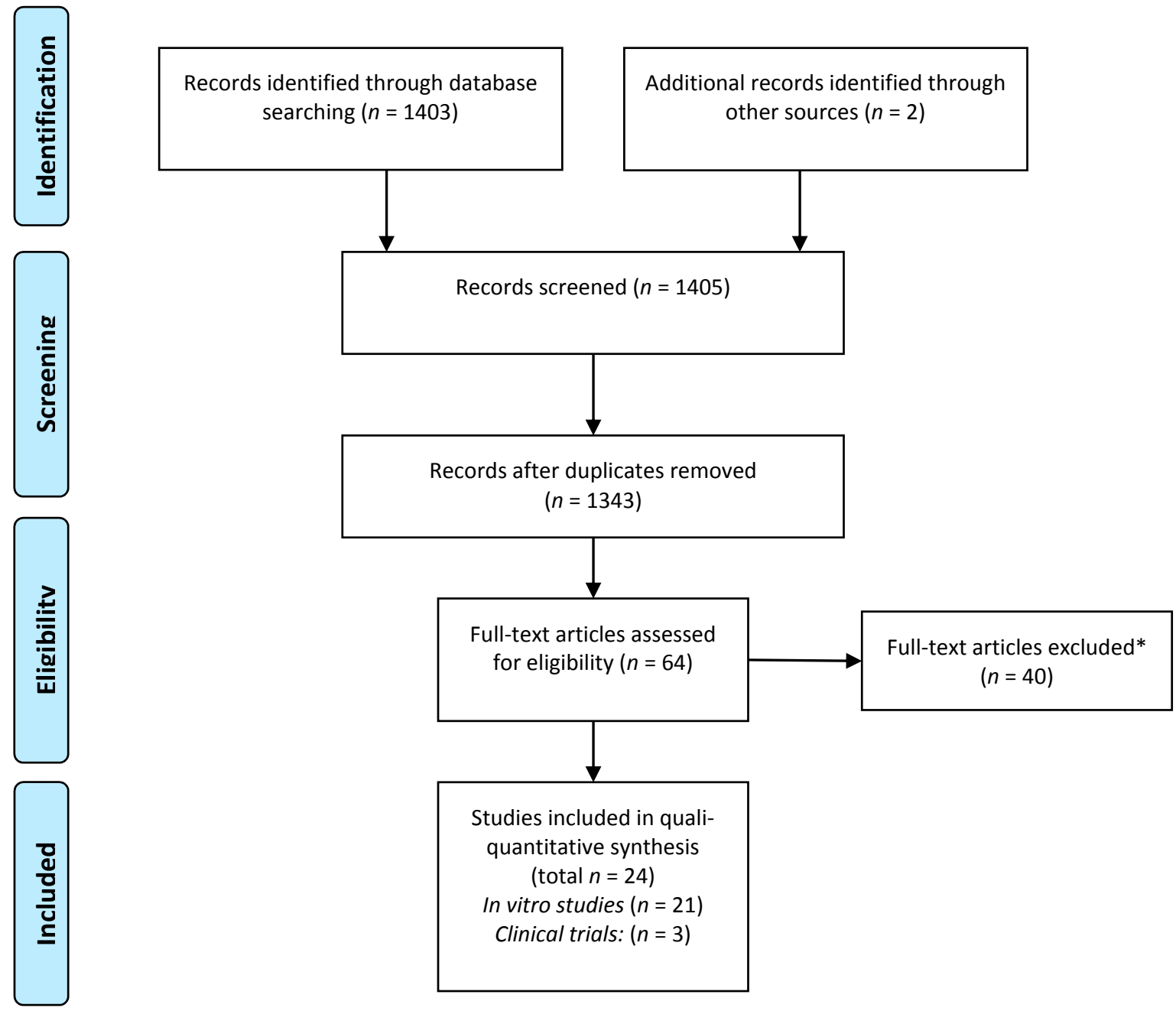

Figure 1. Flow diagram of the search strategy comprising the identification of potentially relevant material, and preliminary screening and final selection of the studies included in this review (based on PRISMA guidelines). * The leading reasons for exclusion of articles were: clinical trials_- "score lower than 3 in Jadad's scale" (see Methods); in vitro studies - lack of critical information on chemical profiling, and methodological shortcomings.

\subsection{In Vitro Studies}

According to the in vitro studies analyzed, there was a predominance of tests with planktonic cultures (Tables 1-6) rather than mono- or multi-species biofilm cultures (Table 7). Of the 22 studies, $5(22.72 \%)$ tested the effect of the EO on streptococci and lactobacilli biofilms. 


\subsubsection{Planktonic Studies}

\section{Crude EOs and Planktonic S. mutans}

Thirty species were found to have very strong or strong antibacterial activity against $S$. mutans, of which the most promising were Achillea ligustica All. (ligurian yarrow) [19], Cryptomeria japonica D. Don (sugi) [20], Croton cajucara Benth (sacaca) [21], Baccharis dracunculifolia DC (broom weed), Coriandrum sativum L. (coriander), Lippia sidoides Cham. (rosemary-pepper), Mikania glomerata Sprengel (guaco) and Siparuna guianenses Aubl. (wild lemon) [11], with planktonic MIC values equal to or lower than $100 \mu \mathrm{g} / \mathrm{mL}$ (Table 1).

Table 1. In vitro antibacterial activity of essential oils against Streptococcus mutans.

\begin{tabular}{|c|c|c|c|c|c|c|}
\hline Plant Species & Source & Microorganism & MIC $(\mu \mathrm{g} / \mathrm{mL})$ & $\operatorname{MBC}(\mu \mathrm{g} / \mathrm{mL})$ & Score & Ref. \\
\hline Achillea ligustica All. & Inflorescences & DSM 20523 & 155 & $\mathrm{nt}$ & +++ & [19] \\
\hline Achillea ligustica All. & Leaves & DSM 20523 & 155 & $\mathrm{nt}$ & +++ & [19] \\
\hline Achillea ligustica All. & flowering aerial parts & DSM 20523 & 38 & nt & ++++ & [19] \\
\hline Achillea ligustica All. & Flowers & DSM 20523 & 155 & 310 & +++ & [22] \\
\hline Achillea ligustica All. & vegetative parts & DSM 20523 & 39 & 39 & ++++ & [22] \\
\hline Ageratum conyzoides & Leaves & ATCC 25175 & 4000 & $\mathrm{nt}$ & - & [23] \\
\hline Aloysia gratissima & Leaves & UA 159 & $125-250$ & $250-500$ & +++ & [11] \\
\hline Aloysia triphylla & Leaves & UA 159 & $125-250$ & $125-250$ & +++ & [11] \\
\hline Alpinia speciosa & Root & UA 159 & $125-250$ & $250-500$ & +++ & [11] \\
\hline Artemisia camphorata Vill. & Leaves & ATCC 25175 & 2000 & nt & + & [23] \\
\hline Baccharis dracunculifolia & Leaves & UA 159 & $62.5-125$ & $250-500$ & ++++ & [11] \\
\hline Bidens sulphurea & Leaves & ATCC 25175 & 250 & $\mathrm{nt}$ & +++ & [23] \\
\hline Cinnamomum zeylanicum & Leaves & UA 159 & $250-500$ & $500-1000$ & +++ & [11] \\
\hline Coriandrum sativum & Leaves & UA 159 & $31.2-62.5$ & $62.5-125$ & ++++ & [11] \\
\hline Croton cajucara Benth & Leaves & ATCC 4646 & 40.1 & 13.8 & ++++ & [21] \\
\hline Cryptomeria japonica & aerial parts & ATCC 25175 & 100 & 200 & ++++ & [20] \\
\hline Cuminum cyminum & CS & PTCC 1601 & 4000 & $\mathrm{nt}$ & - & [24] \\
\hline Cymbopogon citratus & Leaves & UA 159 & $125-250$ & $250-500$ & +++ & [11] \\
\hline Cymbopogon martini & leaves & UA 159 & $125-250$ & $250-500$ & +++ & [11] \\
\hline Cymbopogon winterianus & Leaves & UA 159 & $125-250$ & $250-500$ & +++ & [11] \\
\hline Cyperus articulatus & Bulbs & UA 159 & $125-250$ & $250-500$ & +++ & [11] \\
\hline Elyonurus muticus & Leaves & UA 159 & $125-250$ & $250-500$ & +++ & [11] \\
\hline Eucalyptus radiate & $\mathrm{CS}$ & JC-2 & 10,000 & 10,000 & - & [25] \\
\hline Eugenia caryophyllata L. & $\mathrm{CS}$ & ATCC 25175 & 200 & 800 & +++ & [26] \\
\hline Eugenia caryophyllata L. & $\mathrm{CS}$ & ATCC 5175 & 600 & nt & ++ & [27] \\
\hline Eugenia florida & Leaves & UA 159 & $125-250$ & $125-250$ & +++ & [11] \\
\hline Eugenia uniflora & Leaves & UA 159 & $125-250$ & $250-500$ & +++ & [11] \\
\hline Foeniculum vulgare Mill. & Leaves & ATCC 25175 & $>4000$ & $\mathrm{nt}$ & - & [23] \\
\hline Lavandula officinalis & $\mathrm{CS}$ & $\mathrm{JC}-2$ & $>10,000$ & $>10,000$ & - & [25] \\
\hline Leptosperfum scoparium & $\mathrm{CS}$ & JC-2 & 2500 & 2500 & - & {$[25]$} \\
\hline Lippia alba & Leaves & ATCC 25175 & 500 & $\mathrm{nt}$ & +++ & [23] \\
\hline Lippia alba & Leaves & UA 159 & $125-250$ & $250-500$ & +++ & [11] \\
\hline
\end{tabular}


Table 1. Cont.

\begin{tabular}{|c|c|c|c|c|c|c|}
\hline Plant Species & Source & Microorganism & MIC $(\mu \mathrm{g} / \mathrm{mL})$ & $\operatorname{MBC}(\mu \mathrm{g} / \mathrm{mL})$ & Score & Ref. \\
\hline Lippia sidoides & Leaves & UA 159 & $62.5-125$ & $125-250$ & ++++ & [11] \\
\hline Melaleuca alternifólia & $\mathrm{CS}$ & $\mathrm{JC}-2$ & 10,000 & 10,000 & - & {$[25]$} \\
\hline Melaleuca alternifólia & Leaves & clinical isolates & $0.25-2$ & $0.25-2$ & $*$ & {$[28]$} \\
\hline Mentha piperita & Leaves & UA159 & $250-500$ & $250-500$ & +++ & [11] \\
\hline Mentha piperita & $\mathrm{CS}$ & PTCC 1601 & 6000 & $\mathrm{nt}$ & + & [24] \\
\hline Mikania glomerata & Leaves & UA 159 & $62.5-125$ & $125-250$ & ++++ & [11] \\
\hline Ocimum americanum $\mathrm{L}$. & Leaves & ATCC 6363 & 0.04 & 0.08 & $*$ & [29] \\
\hline Ocimum gratissimum L. & Leaves & ATCC 25175 & 1000 & nt & ++ & {$[23]$} \\
\hline Pelargonium graveolens & Leaves & ATCC 25175 & 1000 & nt & ++ & [23] \\
\hline Romarinus officinalis L. & Leaves & JC-2 & $>10,000$ & $>10,000$ & - & {$[25]$} \\
\hline Rosmarinus officinalis L. & Leaves & ATCC 25275 & $>2000$ & $\mathrm{nt}$ & - & [15] \\
\hline Rosmarinus officinalis L. & $\mathrm{CS}$ & PTCC 1601 & 2000 & $\mathrm{nt}$ & - & {$[30]$} \\
\hline Satureja biflora & flowering aerial parts & clinical isolates & 640 & $\mathrm{nt}$ & ++ & {$[31]$} \\
\hline Satureja masukensis & flowering aerial parts & clinical isolates & 570 & $\mathrm{nt}$ & ++ & [31] \\
\hline Satureja pseudosimensis & $\begin{array}{c}\text { Leaves and flowering } \\
\text { tops }\end{array}$ & clinical isolates & 920 & $\mathrm{nt}$ & ++ & [31] \\
\hline Siparuna guianenses & Leaves & UA 159 & $62.5-125$ & $125-250$ & ++++ & {$[11]$} \\
\hline Syzygium aromaticum & Leaves & ATCC 25175 & 2000 & nt & + & {$[23]$} \\
\hline Syzygium aromaticum & Leaves & UA 159 & $250-500$ & $250-500$ & +++ & [11] \\
\hline Tagetes erecta L. & Leaves & ATCC 25175 & $>4000$ & $\mathrm{nt}$ & - & {$[23]$} \\
\hline Thymus eriocalyx & $\mathrm{CS}$ & PTCC 1601 & 2000 & $\mathrm{nt}$ & + & [30] \\
\hline Zivuphus zoazeiro & Leaves & UA 159 & $250-500$ & $500-1000$ & +++ & [11] \\
\hline
\end{tabular}

Note: * values are expressed as $v / v$; CS (commercial source); nt (not tested); Comparative MIC values: $(++++) \leq 100 ;(+++) 101$ to $500 ;(++) 501$ to $1000 ;(+)>1001$ to $2000 ;(-)>2001$.

Crude EOs and Planktonic S. sobrinus, S. sanguinis and S. salivarius

Four plant species were found to have very strong or strong antibacterial activity against $S$. sobrinus, as follows: Croton cajucara Benth (sacaca) [21]; Rosmarinus officinalis L. (rosemary) [15]; Eugenia caryophyllata L. (clove) [26] and Cryptomeria japonica (sugi) [20]. Of these, C. japonica also had very strong and strong activity against $S$. sanguinis and $S$. salivarius, respectively (Table 2).

Crude EOs and Planktonic Lactobacilli

Achillea ligustica (ligurian yarrow) [19] had strong activity against L. acidophilus. Another species of Lactobacillus, L. casei, was found to be strongly susceptible to Croton cajucara (sacaca) [21], Artemisia camphorata Vill. (camphor), Bidens sulphurea Sch. Bip. (yellow cosmos), Lippia alba Mill. (lemon balm) and Ocimum gratissimum L. (tree basil) [23] (Table 3).

EO-Isolated Compounds against Streptococci and Lactobacilli

Menthol, isolated from Mentha longifolia L., and eugenol, isolated from Eugenia caryophyllata L., were found to be the most promising compounds with strong activity against streptococci and lactobacilli (Tables 4-6). 
Table 2. In vitro antibacterial activity of essential oils against $S$. sobrinus, $S$. sanguinis and $S$. salivarius.

\begin{tabular}{|c|c|c|c|c|c|c|c|c|c|c|c|c|}
\hline \multirow[b]{2}{*}{ Plant Species } & \multirow[b]{2}{*}{ Source } & \multirow[b]{2}{*}{ Microorg } & \multicolumn{3}{|c|}{ S. sobrinus ${ }^{1}$} & \multicolumn{3}{|c|}{ S. sanguinis ${ }^{2}$} & \multicolumn{3}{|c|}{ S. salivarius ${ }^{3}$} & \multirow[b]{2}{*}{ Ref } \\
\hline & & & $\begin{array}{c}\text { MIC } \\
(\mu \mathrm{g} / \mathrm{mL})\end{array}$ & $\begin{array}{c}\text { MBC } \\
(\mu \mathrm{g} / \mathrm{mL})\end{array}$ & Score & $\begin{array}{c}\text { MIC } \\
(\mu \mathrm{g} / \mathrm{mL})\end{array}$ & $\begin{array}{c}\mathrm{MBC} \\
(\mu \mathrm{g} / \mathrm{mL})\end{array}$ & Score & $\begin{array}{c}\text { MIC } \\
(\mu \mathrm{g} / \mathrm{mL})\end{array}$ & $\begin{array}{c}\text { MBC } \\
(\mu \mathrm{g} / \mathrm{mL})\end{array}$ & Score & \\
\hline Achillea ligustica All & inflorescences & $\mathrm{IMC} 04^{3}$ & $\mathrm{nt}$ & $\mathrm{nt}$ & & nt & $\mathrm{nt}$ & & 1250 & $\mathrm{nt}$ & + & [19] \\
\hline Achillea ligustica All & Leaves & IMC104 ${ }^{3}$ & nt & nt & & $\mathrm{nt}$ & nt & & 1250 & nt & + & [19] \\
\hline Achillea ligustica All & flowering aerial parts & $\mathrm{IMC} 104^{3}$ & $\mathrm{nt}$ & nt & & nt & nt & & 625 & $\mathrm{nt}$ & ++ & [19] \\
\hline Ageratum conyzoides $\mathrm{L}$. & Leaves & $\begin{array}{l}\text { ATCC } 33478^{1} \\
\text { ATCC } 10556^{2} \\
\text { ATCC } 25975^{3}\end{array}$ & $>4000$ & nt & - & $>4000$ & nt & - & 4000 & nt & - & [23] \\
\hline Artemisia camphorata Vill. & Leaves & $\begin{array}{l}\text { ATCC } 33478^{1} \\
\text { ATCC } 10556^{2} \\
\text { ATCC } 25975^{3}\end{array}$ & 2000 & nt & + & 2000 & nt & + & 4000 & nt & - & [23] \\
\hline Bidens sulphurea & Leaves & $\begin{array}{l}\text { ATCC } 33478^{1} \\
\text { ATCC } 10556^{2} \\
\text { ATCC } 25975^{3}\end{array}$ & 4000 & nt & - & 4000 & nt & - & 4000 & nt & - & [23] \\
\hline Croton cajucara Benth & Leaves & ATCC $27609^{1}$ & 13.8 & nt & ++++ & nt & $\mathrm{nt}$ & & $\mathrm{nt}$ & nt & & [21] \\
\hline Cryptomeria japonica & aerial parts & $\begin{array}{l}\text { ATCC } 27607^{1} \\
\text { ATCC } 10556^{2}\end{array}$ & 100 & 100 & ++++ & 100 & 200 & ++++ & nt & nt & & [20] \\
\hline Eucalyptus radiate & $\mathrm{CS}$ & $\begin{array}{l}\text { ATCC } 6715^{1} \\
\text { ATCC B13 }^{1}\end{array}$ & 10,000 & 10,000 & - & $\mathrm{nt}$ & $\mathrm{nt}$ & & nt & nt & & [25] \\
\hline Eugenia caryophyllata L. & Flowers & $\begin{array}{l}\text { ATCC } 27607^{1} \\
\text { ATCC } 10556^{2}\end{array}$ & 200 & 800 & +++ & 400 & 800 & +++ & nt & nt & & [26] \\
\hline Foeniculum vulgare Mill. & Leaves & $\begin{array}{l}\text { ATCC } 33478^{1} \\
\text { ATCC } 10556^{2} \\
\text { ATCC } 25975^{3}\end{array}$ & $>4000$ & nt & - & $>4000$ & nt & - & $>4000$ & nt & - & [23] \\
\hline Lavandula officinalis & $\mathrm{CS}$ & $\begin{array}{l}6715^{1} \\
\text { B13 }\end{array}$ & $\begin{array}{l}10,000 \\
10,000\end{array}$ & $\begin{array}{l}10,000 \\
10,000\end{array}$ & $\begin{array}{l}- \\
-\end{array}$ & nt & nt & & $\mathrm{nt}$ & nt & & [25] \\
\hline Leptosperfum scoparium & $\mathrm{CS}$ & $\begin{array}{l}6715^{1} \\
\mathrm{~B} 13^{1}\end{array}$ & $\begin{array}{l}1300 \\
2500\end{array}$ & $\begin{array}{l}2500 \\
2500\end{array}$ & $\begin{array}{l}+ \\
-\end{array}$ & nt & nt & & nt & nt & & [25] \\
\hline
\end{tabular}


Table 2. Cont.

\begin{tabular}{|c|c|c|c|c|c|c|c|c|c|c|c|c|}
\hline \multirow[b]{2}{*}{ Plant Species } & \multirow[b]{2}{*}{ Source } & \multirow[b]{2}{*}{ Microorg } & \multicolumn{3}{|c|}{ S. sobrinus ${ }^{1}$} & \multicolumn{3}{|c|}{ S. sanguinis ${ }^{2}$} & \multicolumn{3}{|c|}{ S. salivarius ${ }^{3}$} & \multirow[b]{2}{*}{ Ref. } \\
\hline & & & $\begin{array}{c}\text { MIC } \\
(\mu \mathrm{g} / \mathrm{mL})\end{array}$ & $\begin{array}{c}\text { MBC } \\
(\mu \mathrm{g} / \mathrm{mL})\end{array}$ & Score & $\begin{array}{c}\text { MIC } \\
(\mu \mathrm{g} / \mathrm{mL})\end{array}$ & $\begin{array}{c}\text { MBC } \\
(\mu \mathrm{g} / \mathrm{mL})\end{array}$ & Score & $\begin{array}{c}\text { MIC } \\
(\mu \mathrm{g} / \mathrm{mL})\end{array}$ & $\begin{array}{c}\text { MBC } \\
(\mu \mathrm{g} / \mathrm{mL})\end{array}$ & Score & \\
\hline Lippia alba & Leaves & $\begin{array}{l}\text { ATCC } 33478^{1} \\
\text { ATCC } 10556^{2} \\
\text { ATCC } 25975^{3}\end{array}$ & 1000 & $\mathrm{nt}$ & ++ & 1000 & $\mathrm{nt}$ & ++ & 2000 & $\mathrm{nt}$ & + & [23] \\
\hline Melaleuca alternifólia & $\mathrm{CS}$ & $\begin{array}{l}6715^{1} \\
\text { B13 }\end{array}$ & $\begin{array}{c}10,000 \\
2500\end{array}$ & $\begin{array}{l}10,000 \\
10,000\end{array}$ & - & $\mathrm{nt}$ & $\mathrm{nt}$ & & $\mathrm{nt}$ & $\mathrm{nt}$ & & [25] \\
\hline Mentha piperita & $\mathrm{CS}$ & $\begin{array}{l}\text { Ssb } 176^{1} \\
\text { Ssg } 009^{2}\end{array}$ & 3000 & $\mathrm{nt}$ & - & 6000 & $\mathrm{nt}$ & - & $\mathrm{nt}$ & $\mathrm{nt}$ & & [32] \\
\hline Ocimum basilicum & $\mathrm{CS}$ & $\begin{array}{l}\text { Ssb } 176^{1} \\
\text { Ssg } 009^{2}\end{array}$ & 6000 & $\mathrm{nt}$ & - & 6000 & $\mathrm{nt}$ & - & $\mathrm{nt}$ & $\mathrm{nt}$ & & [32] \\
\hline Ocimum gratissimum $\mathrm{L}$. & Leaves & $\begin{array}{l}\text { ATCC } 33478^{1} \\
\text { ATCC } 10556^{2} \\
\text { ATCC } 25975^{3}\end{array}$ & 1000 & $\mathrm{nt}$ & ++ & 2000 & $\mathrm{nt}$ & + & 2000 & $\mathrm{nt}$ & + & [23] \\
\hline Pelargonium graveolens & Leaves & $\begin{array}{l}\text { ATCC } 33478^{1} \\
\text { ATCC } 10556^{2} \\
\text { ATCC } 25975^{3}\end{array}$ & 1000 & $\mathrm{nt}$ & ++ & 2000 & $\mathrm{nt}$ & + & 2000 & $\mathrm{nt}$ & + & [23] \\
\hline Rosmarinus officinalis L. & Leaves & $\begin{array}{l}6715^{1} \\
\text { B13 }\end{array}$ & $\begin{array}{l}10,000 \\
10,000\end{array}$ & $\begin{array}{l}10,000 \\
10,000\end{array}$ & $\begin{array}{l}- \\
-\end{array}$ & nt & $\mathrm{nt}$ & & $\mathrm{nt}$ & $\mathrm{nt}$ & & [25] \\
\hline Rosmarinus officinalis L. & Leaves & $\begin{array}{l}\text { ATCC } 33478^{1} \\
\text { ATCC } 10556^{2} \\
\text { ATCC } 25975^{3}\end{array}$ & 500 & $\mathrm{nt}$ & +++ & $>2000$ & $\mathrm{nt}$ & - & 600 & $\mathrm{nt}$ & ++ & [15] \\
\hline Salvia officinalis & $\mathrm{CS}$ & $\begin{array}{l}\text { Ssb } 176^{1} \\
\text { Ssg } 009^{2}\end{array}$ & 3000 & $\mathrm{nt}$ & - & 6000 & $\mathrm{nt}$ & - & $\mathrm{nt}$ & $\mathrm{nt}$ & & [32] \\
\hline Syzygium aromaticum & Leaves & $\begin{array}{l}\text { ATCC } 33478^{1} \\
\text { ATCC } 10556^{2} \\
\text { ATCC } 25975^{3} \\
\end{array}$ & $>4000$ & $\mathrm{nt}$ & - & $>4000$ & nt & - & $>4000$ & nt & - & [23] \\
\hline Tagetes erecta $\mathrm{L}$. & Leaves & $\begin{array}{l}\text { Ssb } 176^{1} \\
\text { Ssg } 009^{2} \\
\end{array}$ & 6000 & $\mathrm{nt}$ & - & $\mathrm{nt}$ & $\mathrm{nt}$ & & $\mathrm{nt}$ & $\mathrm{nt}$ & & [32] \\
\hline
\end{tabular}

Note: $\mathrm{CS}=$ commercial source; $\mathrm{nt}$ (not tested); Comparative MIC values: $(++++) \leq 100 ;(+++) 101$ to $500 ;(++) 501$ to $1000 ;(+)>1001$ to $2000 ;(-)>2001 ;{ }^{1}$ S. sobrinus;

${ }^{2} S$. sanguinis and ${ }^{3}$ S. salivarius. 
Table 3. In vitro antibacterial activity of essential oils against lactobacilli.

\begin{tabular}{|c|c|c|c|c|c|c|c|c|c|}
\hline \multirow[b]{2}{*}{ Plant Species } & \multirow[b]{2}{*}{ Source } & \multirow[b]{2}{*}{ Microorg } & \multicolumn{3}{|c|}{ L. acidophilus ${ }^{1}$} & \multicolumn{3}{|c|}{ L. casei $^{2}$} & \multirow[b]{2}{*}{ Ref. } \\
\hline & & & $\begin{array}{c}\text { MIC } \\
(\mu \mathrm{g} / \mathrm{mL})\end{array}$ & $\begin{array}{c}\text { MBC } \\
(\mu \mathrm{g} / \mathrm{mL})\end{array}$ & Score & $\begin{array}{c}\text { MIC } \\
(\mu \mathrm{g} / \mathrm{mL})\end{array}$ & $\begin{array}{c}\text { MBC } \\
(\mu \mathrm{g} / \mathrm{mL})\end{array}$ & Score & \\
\hline Achillea ligustica All. & Inflorescences & $\operatorname{IMC} 101^{1}$ & 310 & nt & +++ & nt & nt & & [19] \\
\hline Achillea ligustica All. & Leaves & $\operatorname{IMC} 101^{1}$ & 2500 & $\mathrm{nt}$ & - & $\mathrm{nt}$ & $\mathrm{nt}$ & & [19] \\
\hline Achillea ligustica All. & flowering aerial parts & IMC $101^{1}$ & 1250 & $\mathrm{nt}$ & + & $\mathrm{nt}$ & $\mathrm{nt}$ & & [19] \\
\hline Ageratum conyzoides L. & Leaves & ATCC $11578^{2}$ & $\mathrm{nt}$ & $\mathrm{nt}$ & & 4000 & $\mathrm{nt}$ & - & [23] \\
\hline Artemisia camphorata Vill. & Leaves & ATCC $11578^{2}$ & $\mathrm{nt}$ & $\mathrm{nt}$ & & 500 & $\mathrm{nt}$ & +++ & [23] \\
\hline Bidens sulphurea & Leaves & ATCC $11578^{2}$ & $\mathrm{nt}$ & $\mathrm{nt}$ & & 500 & $\mathrm{nt}$ & +++ & [23] \\
\hline Croton cajucara Benth & Leaves & ATCC $4646^{2}$ & $\mathrm{nt}$ & $\mathrm{nt}$ & & 22.3 & $\mathrm{nt}$ & ++++ & [21] \\
\hline Foeniculum vulgare Mill. & Leaves & ATCC $11578^{2}$ & $\mathrm{nt}$ & $\mathrm{nt}$ & & 4000 & $\mathrm{nt}$ & - & [23] \\
\hline Lippia alba & Leaves & ATCC $11578^{2}$ & $\mathrm{nt}$ & $\mathrm{nt}$ & & 500 & $\mathrm{nt}$ & +++ & [23] \\
\hline Ocimum americanum $\mathrm{L}$. & Leaves & ATCC $6363^{2}$ & $\mathrm{nt}$ & $\mathrm{nt}$ & & 0.04 & $0.3 *$ & $*$ & [29] \\
\hline Ocimum basilicum & aerial parts & $\operatorname{ATCC} 4356^{1}$ & 80,000 & $\mathrm{nt}$ & - & $\mathrm{nt}$ & $\mathrm{nt}$ & & [33] \\
\hline Ocimum gratissimum $\mathrm{L}$. & Leaves & ATCC $11578^{2}$ & $\mathrm{nt}$ & $\mathrm{nt}$ & & 500 & $\mathrm{nt}$ & +++ & [23] \\
\hline Origanum vulgare & aerial parts & ATCC $4356^{1}$ & 5000 & $\mathrm{nt}$ & - & $\mathrm{nt}$ & $\mathrm{nt}$ & & [33] \\
\hline Pelargonium graveolens & Leaves & ATCC $11578^{2}$ & $\mathrm{nt}$ & $\mathrm{nt}$ & & 1000 & $\mathrm{nt}$ & ++ & [23] \\
\hline Rosmarinus officinalis & aerial parts & ATCC $4356^{1}$ & 80,000 & $\mathrm{nt}$ & - & $\mathrm{nt}$ & $\mathrm{nt}$ & & [33] \\
\hline Salvia officinalis & aerial parts & ATCC $4356^{1}$ & 80,000 & $\mathrm{nt}$ & - & $\mathrm{nt}$ & $\mathrm{nt}$ & & [33] \\
\hline Syzygium aromaticum & Leaves & ATCC $11578^{2}$ & $\mathrm{nt}$ & $\mathrm{nt}$ & & 1000 & $\mathrm{nt}$ & ++ & [23] \\
\hline Tagetes erecta $\mathrm{L}$. & Leaves & ATCC $11578^{2}$ & $\mathrm{nt}$ & $\mathrm{nt}$ & & 4000 & $\mathrm{nt}$ & - & [23] \\
\hline Thymus vulgaris & aerial parts & ATCC $4356^{1}$ & 5000 & $\mathrm{nt}$ & - & nt & $\mathrm{nt}$ & & [33] \\
\hline
\end{tabular}

Note: * values are expressed as $\%(v / v)$; nt (not tested); Comparative MIC values: $(++++)<100 ;(+++) 100$ to 500; $(++) 501$ to $1000 ;(+)>1001$ to $2000 ;(-)>2001 ;{ }^{1}$ L. acidophilus $;{ }^{2}$ L. casei.

Table 4. Essential oils isolated compounds against Streptococcus mutans.

\begin{tabular}{|c|c|c|c|c|c|c|}
\hline Compound & Plant Species & $\begin{array}{c}\text { Culture } \\
\text { Collection }\end{array}$ & $\begin{array}{c}\text { MIC } \\
(\mu \mathrm{g} / \mathrm{mL})\end{array}$ & $\begin{array}{c}\text { MBC } \\
(\mu \mathrm{g} / \mathrm{mL})\end{array}$ & Score & Ref. \\
\hline 1,8, Cineole & Achillea ligustica All & DSM 20523 & 2500 & $\mathrm{nt}$ & - & [19] \\
\hline 1,8, Cineole & Achillea ligustica All & DSM 20523 & 155 & 1250 & +++ & [22] \\
\hline 1,8, Cineole & Rosmarinus officinalis & ATCC 25275 & 1500 & nt & + & [15] \\
\hline Camphor & Rosmarinus officinalis & ATCC 25275 & 1500 & $\mathrm{nt}$ & + & {$[15]$} \\
\hline Caryophyllene oxide & Satureja species & clinical isolates & 250 & $\mathrm{nt}$ & +++ & [31] \\
\hline Eugenol & Eugenia caryophyllata L. & ATCC 25175 & 100 & 200 & ++++ & [26] \\
\hline Linalool & Achillea ligustica All & DSM 20523 & 625 & $\mathrm{nt}$ & ++ & [19] \\
\hline Linalool & Achillea ligustica All & DSM 20523 & 310 & 310 & +++ & [22] \\
\hline Linalool & Croton cajucara Benth & ATCC 25175 & no activity & $\mathrm{nt}$ & - & [21] \\
\hline Linalool & Satureja species & clinical isolates & 370 & $\mathrm{nt}$ & +++ & [31] \\
\hline Menthol & Mentha longifolia L. & clinical isolates & 15.6 & $\mathrm{nt}$ & ++++ & [34] \\
\hline Pulegone & Satureja species & clinical isolates & 1750 & $\mathrm{nt}$ & + & [31] \\
\hline Sabinene & Cryptomeria japonica & ATCC 25175 & 800 & 1600 & ++ & [20] \\
\hline Terpinen-4-ol & Achillea ligustica All & DSM 20523 & 1250 & $\mathrm{nt}$ & + & [19] \\
\hline Terpinen-4-ol & Achillea ligustica All & DSM 20523 & 310 & 625 & +++ & [22] \\
\hline Terpinen-4-ol & Cryptomeria japonica & ATCC 25175 & 1600 & 3200 & + & [20] \\
\hline Verbenone & Rosmarinus officinalis & ATCC 25275 & 1000 & $\mathrm{nt}$ & ++ & [15] \\
\hline
\end{tabular}


Table 4. Cont.

\begin{tabular}{lcccccc}
\hline Compound & Plant Species & $\begin{array}{c}\text { Culture } \\
\text { Collection }\end{array}$ & $\begin{array}{c}\text { MIC } \\
(\boldsymbol{\mu g} / \mathbf{m L})\end{array}$ & $\begin{array}{c}\text { MBC } \\
(\boldsymbol{\mu g} / \mathbf{m L})\end{array}$ & Score & Ref. \\
\hline Viridiflorol & Achillea ligustica All & DSM 20523 & 2500 & $\mathrm{nt}$ & - & {$[19]$} \\
$\alpha$-Pinene & Cryptomeria japonica & ATCC 25175 & 6400 & 28,000 & - & {$[20]$} \\
$\alpha$-Pinene & Rosmarinus officinalis & ATCC 25275 & 2000 & $\mathrm{nt}$ & + & {$[15]$} \\
$\alpha$-Terpineol & Cryptomeria japonica & ATCC 25175 & 1600 & 3200 & + & {$[20]$} \\
$\beta$-Caryophyllene & Eugenia caryophyllata L. & ATCC 25175 & 1600 & 3200 & + & {$[26]$} \\
$\beta$-Caryophyllene & Rosmarinus officinalis & ATCC 25275 & 300 & $\mathrm{nt}$ & +++ & {$[15]$} \\
$\beta$-Myrcene & Rosmarinus officinalis & ATCC 25275 & 400 & $\mathrm{nt}$ & +++ & {$[15]$} \\
$\beta$-Pinene & Achillea ligustica All & DSM 20523 & 1250 & $\mathrm{nt}$ & + & {$[19]$} \\
$\beta$-Pinene & Achillea ligustica All & DSM 20523 & 625 & 1250 & ++ & {$[22]$} \\
$\gamma$-Terpinene & Achillea ligustica All & DSM 20523 & 2500 & $\mathrm{nt}$ & - & {$[19]$} \\
\hline
\end{tabular}

Note: CS (commercial source); nt (not tested); Comparative MIC values: $(++++)<100$; (+++) 100 to 500; (++) 501 to $1000 ;(+)>1001$ to $2000 ;(-)>2001$.

Table 5. Essential oils isolated compounds against lactobacilli.

\begin{tabular}{|c|c|c|c|c|c|c|c|c|c|}
\hline \multirow[b]{2}{*}{ Compound } & \multirow[b]{2}{*}{ Source } & \multirow{2}{*}{$\begin{array}{c}\text { Culture } \\
\text { Collection }\end{array}$} & \multicolumn{3}{|c|}{ L. acidophilus ${ }^{1}$} & \multicolumn{3}{|c|}{ L. casei $^{2}$} & \multirow[b]{2}{*}{ Ref. } \\
\hline & & & $\begin{array}{c}\text { MIC } \\
(\mu \mathrm{g} / \mathrm{mL})\end{array}$ & $\begin{array}{c}\text { MBC } \\
(\mu \mathrm{g} / \mathrm{mL})\end{array}$ & Score & $\begin{array}{c}\text { MIC } \\
(\mu \mathrm{g} / \mathrm{mL}) \\
\end{array}$ & $\begin{array}{c}\text { MBC } \\
(\mu \mathrm{g} / \mathrm{mL})\end{array}$ & Score & \\
\hline 1,8, Cineole * & Achillea ligustica All & $\mathrm{IMC} 101^{1}$ & 5000 & $\mathrm{nt}$ & - & nt & $\mathrm{nt}$ & & [19] \\
\hline Linalool & Croton cajucara Benth & ATCC $4646^{2}$ & $\mathrm{nt}$ & $\mathrm{nt}$ & & no activity & $\mathrm{nt}$ & - & [21] \\
\hline Linalool * & Achillea ligustica All & $\mathrm{IMC} 101^{1}$ & 5000 & $\mathrm{nt}$ & - & $\mathrm{nt}$ & $\mathrm{nt}$ & & [19] \\
\hline Menthol & Mentha longifolia L. & clinical isolates & 31.2 & $\mathrm{nt}$ & ++++ & $\mathrm{nt}$ & $\mathrm{nt}$ & & [34] \\
\hline Terpinen-4-ol * & Achillea ligustica All & $\mathrm{IMC} 101^{1}$ & 5000 & $\mathrm{nt}$ & - & $\mathrm{nt}$ & $\mathrm{nt}$ & & [19] \\
\hline$\beta$-Pinene * & Achillea ligustica All & $\mathrm{IMC} 01^{1}$ & 2500 & nt & - & $\mathrm{nt}$ & $\mathrm{nt}$ & & [19] \\
\hline$\gamma$-Terpinene $*$ & Achillea ligustica All & $\mathrm{IMC} 101^{1}$ & 5000 & nt & - & nt & $\mathrm{nt}$ & & [19] \\
\hline
\end{tabular}

Note: * standard from Sigma-Aldrich ${ }^{\circledR}$ (St. Louis, MO, USA); nt (not tested); Comparative MIC values: $(++++)<100 ;(-)>2001 ;{ }^{1}$ L. acidophilus ${ }^{2}$ L. casei. 
Table 6. Essential oils isolated compounds against $S$. sobrinus, S. sanguinis and S. salivarius.

\begin{tabular}{|c|c|c|c|c|c|c|c|c|c|c|c|c|}
\hline \multirow[b]{2}{*}{ Compound } & \multirow[b]{2}{*}{ Plant Species } & \multirow{2}{*}{$\begin{array}{l}\text { Culture } \\
\text { Collection }\end{array}$} & \multicolumn{3}{|c|}{ S. sobrinus ${ }^{1}$} & \multicolumn{3}{|c|}{ S. sanguinis ${ }^{2}$} & \multicolumn{3}{|c|}{ S. salivarius ${ }^{3}$} & \multirow[b]{2}{*}{ Ref. } \\
\hline & & & $\begin{array}{c}\text { MIC } \\
(\mu \mathrm{g} / \mathrm{mL})\end{array}$ & $\begin{array}{c}\text { MBC } \\
(\mu \mathrm{g} / \mathrm{mL})\end{array}$ & Score & $\begin{array}{c}\text { MIC } \\
(\mu \mathrm{g} / \mathrm{mL})\end{array}$ & $\begin{array}{c}\text { MBC } \\
(\mu \mathrm{g} / \mathrm{mL})\end{array}$ & Score & $\begin{array}{c}\text { MIC } \\
(\mu \mathrm{g} / \mathrm{mL})\end{array}$ & $\begin{array}{c}\text { MBC } \\
(\mu \mathrm{g} / \mathrm{mL})\end{array}$ & Score & \\
\hline 1,8-cineole & Achillea ligustica All & $\mathrm{IMC} 104^{3}$ & $\mathrm{nt}$ & $\mathrm{nt}$ & & $\mathrm{nt}$ & $\mathrm{nt}$ & & 1250 & $\mathrm{nt}$ & + & [19] \\
\hline Camphor & Rosmarinus officinalis & $\begin{array}{l}\text { ATCC } 33478^{1} \\
\text { ATCC } 10556^{2} \\
\text { ATCC } 25975^{3}\end{array}$ & 1500 & $\mathrm{nt}$ & + & 400 & $\mathrm{nt}$ & +++ & 400 & $\mathrm{nt}$ & +++ & {$[15]$} \\
\hline Eugenol & Eugenia caryophyllata L. & $\begin{array}{l}\text { ATCC } 27607^{1} \\
\text { ATCC } 10556^{2}\end{array}$ & 200 & 400 & +++ & 400 & 800 & +++ & $\mathrm{nt}$ & $\mathrm{nt}$ & & {$[26]$} \\
\hline Linalool & Achillea ligustica All & $\mathrm{IMC} 104^{3}$ & $\mathrm{nt}$ & $\mathrm{nt}$ & & nt & $\mathrm{nt}$ & & 625 & $\mathrm{nt}$ & ++ & [19] \\
\hline Linalool & Croton cajucara Benth & ATCC $27609^{1}$ & no activity & $\mathrm{nt}$ & - & $\mathrm{nt}$ & $\mathrm{nt}$ & & $\mathrm{nt}$ & $\mathrm{nt}$ & & [21] \\
\hline Sabinene & Cryptomeria japonica & $\begin{array}{l}\text { ATCC } 27607^{1} \\
\text { ATCC } 10556^{2}\end{array}$ & 200 & 200 & +++ & 400 & 400 & +++ & nt & $\mathrm{nt}$ & & {$[20]$} \\
\hline Terpinen-4-ol & Achillea ligustica All & $\mathrm{IMC} 104^{3}$ & $\mathrm{nt}$ & $\mathrm{nt}$ & & $\mathrm{nt}$ & $\mathrm{nt}$ & & 625 & $\mathrm{nt}$ & ++ & [19] \\
\hline Terpinen-4-ol & Cryptomeria japônica & $\begin{array}{l}\text { ATCC } 27607^{1} \\
\text { ATCC } 10556^{2}\end{array}$ & 1600 & 3200 & + & 1600 & 3200 & + & $\mathrm{nt}$ & $\mathrm{nt}$ & & {$[20]$} \\
\hline Verbenone & Rosmarinus officinalis & $\begin{array}{l}\text { ATCC } 33478^{1} \\
\text { ATCC } 10556^{2} \\
\text { ATCC } 25975^{3}\end{array}$ & 1000 & $\mathrm{nt}$ & ++ & 400 & $\mathrm{nt}$ & +++ & 400 & $\mathrm{nt}$ & +++ & {$[15]$} \\
\hline Viridiflorol & Achillea ligustica All & IMC104 ${ }^{3}$ & $\mathrm{nt}$ & $\mathrm{nt}$ & & $\mathrm{nt}$ & $\mathrm{nt}$ & & 625 & $\mathrm{nt}$ & ++ & [19] \\
\hline$\alpha$-Pinene & Cryptomeria japonica & $\begin{array}{l}\text { ATCC } 27607^{1} \\
\text { ATCC } 10556^{2}\end{array}$ & 6400 & 12.800 & - & 6400 & 6400 & - & $\mathrm{nt}$ & $\mathrm{nt}$ & & {$[20]$} \\
\hline$\alpha$-Pinene & Rosmarinus officinalis & $\begin{array}{l}\text { ATCC } 33478^{1} \\
\text { ATCC } 10556^{2} \\
\text { ATCC } 25975^{3}\end{array}$ & 1000 & $\mathrm{nt}$ & ++ & 400 & $\mathrm{nt}$ & +++ & 400 & $\mathrm{nt}$ & +++ & {$[15]$} \\
\hline$\alpha$-Terpineol & Cryptomeria japônica & $\begin{array}{l}\text { ATCC } 27607^{1} \\
\text { ATCC } 10556^{2}\end{array}$ & 1600 & 1600 & + & 1600 & 3200 & + & $\mathrm{nt}$ & $\mathrm{nt}$ & & {$[20]$} \\
\hline
\end{tabular}


Table 6. Cont

\begin{tabular}{|c|c|c|c|c|c|c|c|c|c|c|c|c|}
\hline \multirow[b]{2}{*}{ Compound } & \multirow[b]{2}{*}{ Plant Species } & \multirow{2}{*}{$\begin{array}{c}\text { Culture } \\
\text { Collection }\end{array}$} & \multicolumn{3}{|c|}{ S. sobrinus ${ }^{1}$} & \multicolumn{3}{|c|}{ S. sanguinis ${ }^{2}$} & \multicolumn{3}{|c|}{ S. salivarius ${ }^{3}$} & \multirow[b]{2}{*}{ Ref } \\
\hline & & & $\begin{array}{c}\text { MIC } \\
(\mu \mathrm{g} / \mathrm{mL})\end{array}$ & $\begin{array}{c}\text { MBC } \\
(\mu \mathrm{g} / \mathrm{mL})\end{array}$ & Score & $\begin{array}{c}\text { MIC } \\
(\mu \mathrm{g} / \mathrm{mL})\end{array}$ & $\begin{array}{c}\text { MBC } \\
(\mu \mathrm{g} / \mathrm{mL})\end{array}$ & Score & $\begin{array}{c}\text { MIC } \\
(\mu \mathrm{g} / \mathrm{mL})\end{array}$ & $\begin{array}{c}\text { MBC } \\
(\mu \mathrm{g} / \mathrm{mL})\end{array}$ & Score & \\
\hline$\beta$-Caryophyllene & Eugenia caryophyllata L. & $\begin{array}{l}\text { ATCC } 27607^{1} \\
\text { ATCC } 10556^{2}\end{array}$ & 12,800 & 12,800 & - & 1600 & 3200 & + & $\mathrm{nt}$ & $\mathrm{nt}$ & & {$[26]$} \\
\hline$\beta$-Caryophyllene & Rosmarinus officinalis & $\begin{array}{l}\text { ATCC } 33478^{1} \\
\text { ATCC } 10556^{2} \\
\text { ATCC } 25975^{3} \\
\end{array}$ & 400 & $\mathrm{nt}$ & +++ & 400 & $\mathrm{nt}$ & +++ & 400 & $\mathrm{nt}$ & +++ & {$[15]$} \\
\hline$\beta$-Myrcene & Rosmarinus officinalis & $\begin{array}{l}\text { ATCC } 33478^{1} \\
\text { ATCC } 10556^{2} \\
\text { ATCC } 25975^{3}\end{array}$ & 1500 & $\mathrm{nt}$ & + & 1500 & $\mathrm{nt}$ & + & 400 & $\mathrm{nt}$ & +++ & {$[15]$} \\
\hline$\beta$-Pinene & Achillea ligustica All & $\mathrm{IMC} 104^{3}$ & $\mathrm{nt}$ & $\mathrm{nt}$ & & $\mathrm{nt}$ & $\mathrm{nt}$ & & 625 & $\mathrm{nt}$ & ++ & {$[19]$} \\
\hline$\gamma$-Terpinene & Achillea ligustica All & $\mathrm{IMC} 104^{3}$ & $\mathrm{nt}$ & $\mathrm{nt}$ & & $\mathrm{nt}$ & $\mathrm{nt}$ & & 625 & $\mathrm{nt}$ & ++ & [19] \\
\hline
\end{tabular}

Note: $n t$ (not tested); Comparative MIC values: $(+++) 100$ to $500 ;(++) 501$ to $1000 ;(+)>1001$ to $2000 ;(-)>2001 ;{ }^{1}$ S. sobrinus; ${ }^{2}$ S. sanguinis and ${ }^{3}$ S. salivarius. 


\subsubsection{Biofilm Studies}

Crude EOs and Biofilms of Streptococci and Lactobacilli

A total of eight species were tested against biofilm cultures of $S$. mutans, $S$. sobrinus and/or L. casei using different assays (Table 7). Interestingly, bioactive fractions of C. sativum and B. dracunculifolia inhibited $90 \%$ of $S$. mutans biofilm formation at concentrations as low as $31.2 \mu \mathrm{g} / \mathrm{mL}$. Moreover, C. cajucara EO $(100 \mu \mathrm{g} / \mathrm{mL})$ and O. americanum EO (3\%) inhibited S. mutans and L. lactis biofilms as effectively as chlorhexidine, used as positive control.

Overall, the majority of studies in this review tested the effectiveness of EO against $S$. mutans (35 out of 40 studies), followed in lower proportions by $S$. sobrinus, S. salivarius, S. sanguinis and Lactobacillus spp. As seen in Table 8, just a few studies carried out a comprehensive analysis of the effect of EO against a broad panel of caries-related species.

\subsection{In Vivo Studies}

\section{Randomized Clinical Trials}

Three high quality randomized, double-blind clinical trials of herbal interventions with low risk of bias were included in this review (Figure 2). The EOs from L. sidoides [35,36] and a multi-herbal formulation including Melaleuca alternifolia and Leptospermum scoparium oils (combined with Calendula officinalis and Camellia sinensis extracts) [37], were tested in humans for their effectiveness in reducing the amount of cariogenic biofilm, measured by means of plaque indexes. The experimental period of studies ranged from 1 week to 12 weeks, with different assessment checkpoints and dosing protocols. As seen in Table 9, only individuals treated with $1 \%$ L. sidoides EO mouthwash had a statistically significant reduction in their supragingival biofilm levels compared to chlorhexidine group (positive control) and to their baseline condition.

\subsection{Chemical and Botanical Characterization and Georeferencing of the most Promising} Bioactive EOS

Viridiflorol, terpinen-4-ol and $\beta$-pinene are found in the EO from all parts [19,22] of A. lingustica; however, important terpenes such as linalool, 1,8-cineole and germacrene D have also been identified in specific parts of the plant. Elemol, terpinen-4-ol, sabinene, 10(15)-cadinen-4-ol, $\alpha$-terpineol and $\alpha$-pinene are the major compounds identified in C. japonica [20]. Linalool is the most abundant compound of C. cajucara Benth [21]. Trans-nerolidol, spathulenol and trans-caryophyllene are found in B. dracunculifolia [11]. 1-decanol, trans-2-decen-1-ol and 2-dodecen-1-ol are the most abundant compounds of C. sativum [11]. Thymol is the major compound of L. sidoides [11]. Camphor, verbenone, $\alpha$-pinene, $\beta$-myrcene, 1,8-cineole and $\beta$-caryophyllene are found in $R$. officinalis [15]. Eugenol and $\beta$-caryophyllene are the major compounds of E. caryophyllata [26]. 
Table 7. Essential oils, fractions or isolated compounds against in vitro oral biofilm formation.

\begin{tabular}{|c|c|c|c|c|c|}
\hline \multirow{2}{*}{ Ref. } & \multirow{2}{*}{$\begin{array}{l}\text { Essential Oil/Fraction or } \\
\text { Isolated Compound }\end{array}$} & \multicolumn{4}{|c|}{ Biofilm Formation } \\
\hline & & Strain & Test(s) Performed & Biofilm Age Conditions & Outcomes \\
\hline [11] & $\begin{array}{l}\text { Aloysia gratissima }(\mathrm{Ag}) \text {, } \\
\text { Coriandrum sativum }(\mathrm{Cs}) \text { and } \\
\text { Baccharis dracunculifolia }(\mathrm{Bd}) \\
\text { fraction }\end{array}$ & S. mutans UA159 & $\begin{array}{l}\text { Formation of S. mutans biofilm, the } \\
\text { samples were placed in the wells of sterile } \\
\text { polystyrene U-bottom microtiter plates, } \\
\text { previously treated with saliva }\end{array}$ & $\begin{array}{l}\text { S. mutans cells }\left(1.0 \times 10^{7} \text { cells } / \mathrm{mL} \text { in BHI }\right. \\
\text { medium) were added to wells containing BHI } \\
\text { medium with } 2 \% \text { sucrose and the samples } \\
\text { were incubated at } 37^{\circ} \mathrm{C} \text { for } 18 \mathrm{~h}\end{array}$ & $\begin{array}{l}\text { Biofilm of Cs } 4 \text { and } \mathrm{Bd} 2 \text { fractions presented a better } \\
\text { performance since they inhibited more than } 90 \% \text { of } \\
\text { biofilm formation at lower concentrations }(31.2 \mu \mathrm{g} / \mathrm{mL}) \text {. }\end{array}$ \\
\hline [21] & Croton cajucara Benth leaves & S. mutans ATCC 25175 & $\begin{array}{l}\text { Macro technique using microbial disks } \\
\text { subjected to the action of the essential oil } \\
\text { and controls }\end{array}$ & $\begin{array}{l}\text { The biofilms were exposed to controls and } \\
\text { essential oil for } 3 \text { min and incubated for } 72 \mathrm{~h} \\
\text { at } 37^{\circ} \mathrm{C}\end{array}$ & $\begin{array}{l}\text { Growth inhibition: EO } 70 \%-75 \% \\
\text { Chlorhexidine } 65 \%-70 \%\end{array}$ \\
\hline [21] & Croton cajucara Benth leaves & $\begin{array}{l}\text { S. sobrinus } \\
\text { ATCC } 27609\end{array}$ & $\begin{array}{l}\text { Macro technique using microbial disks } \\
\text { subjected to the action of the essential oil } \\
\text { and controls }\end{array}$ & $\begin{array}{l}\text { The biofilms were exposed to controls and } \\
\text { essential oil for } 3 \mathrm{~min} \text { and incubated for } 72 \mathrm{~h} \\
\text { at } 37^{\circ} \mathrm{C}\end{array}$ & $\begin{array}{l}\text { Growth inhibition: EO } 75 \%-80 \% \\
\text { Chlorhexidine } 50 \%-55 \%\end{array}$ \\
\hline [21] & Croton cajucara Benth leaves & L. casei ATCC 4646 & $\begin{array}{l}\text { Macro technique using microbial disks } \\
\text { subjected to the action of the essential oil } \\
\text { and controls }\end{array}$ & $\begin{array}{l}\text { The biofilms were exposed to controls and } \\
\text { essential oil for } 3 \text { min and incubated } \\
\text { for } 72 \mathrm{~h} \text { at } 37^{\circ} \mathrm{C}\end{array}$ & $\begin{array}{l}\text { Growth inhibition: EO } 80 \%-85 \% \\
\text { Chlorhexidine } 65 \%-70 \%\end{array}$ \\
\hline [38] & Curcuma longa root & S. mutans ATCC 25175 & $\begin{array}{l}\text { Technique using 24-well plates containing } \\
\text { resin teeth. }\end{array}$ & $\begin{array}{l}\text { After cultivating S. mutans for } 24 \mathrm{~h} \text { at } 37^{\circ} \mathrm{C} \text {, } \\
\text { the supernatant was removed, and the wells } \\
\text { were rinsed with distilled } \mathrm{H}_{2} \mathrm{O} \text {. Biofilm } \\
\text { formation in the wells was measured by } \\
\text { staining with } 0.1 \% \text { safranin }\end{array}$ & $\begin{array}{l}\text { Biofilm formation was decreased in the presence of } \\
\text { C. longa essential oil at concentrations higher } \\
\text { than } 500 \mu \mathrm{g} / \mathrm{mL}\end{array}$ \\
\hline [39] & $\begin{array}{l}\text { Mentha piperita and } \\
\text { Rosmarinus officinalis }\end{array}$ & S. mutans PTCC 1601 & Biofilm formation (SBF) assay & $\begin{array}{l}\text { The biofilms were exposed to controls } \\
\text { and essential oil and incubated for } 17 \pm 1 \mathrm{~h} \\
\text { at } 37^{\circ} \mathrm{C}\end{array}$ & $\begin{array}{l}\text { M. piperita and } R \text {. officinalis oils effectively inhibited } \\
\text { S. mutans biofilm at } 6000 \text { and } 2000 \mathrm{ppm} \text {, respectively. }\end{array}$ \\
\hline [29] & Ocimum americanum L. leaves & S. mutans KPSK2 & $\begin{array}{l}\text { Microtiter technique Protocol } \\
\text { using saliva. }\end{array}$ & $\begin{array}{l}\text { The biofilms were exposed to controls and } \\
\text { essential oil }(0.3 \% \text { and } 3 \% v / v) \text { for } 5 \mathrm{~min} \text { and } \\
\text { incubated for } 24 \mathrm{~h}\end{array}$ & $\begin{array}{l}\text { EO } 0.3 \%(v / v) 7.2 \times 10^{4} \mathrm{CFU} / \mathrm{mL} ; \text { EO } 3 \%(v / v) \\
2.9 \times 10^{3} \mathrm{CFU} / \mathrm{mL} ; 0.2 \% \text { Chlorhexidine: } \\
1.7 \times 10^{3} \mathrm{CFU} / \mathrm{mL} ; \text { Saline solution } 8.5 .10^{6} \mathrm{CFU} / \mathrm{mL}\end{array}$ \\
\hline [29] & Ocimum americanum $\mathrm{L}$. leaves & L. casei ATCC 6363 & Microtiter technique Protocol using saliva. & $\begin{array}{l}\text { The biofilms were exposed to controls and } \\
\text { essential oil }(0.3 \% \text { and } 3 \% \mathrm{v} / \mathrm{v}) \text { for } 5 \mathrm{~min} \text { and } \\
\text { incubated for } 24 \mathrm{~h}\end{array}$ & $\begin{array}{l}\text { EO } 0.3 \%(v / v) 5.1 \times 10^{5} \mathrm{CFU} / \mathrm{mL} ; \text { EO } 3 \%(v / v) \\
6.3 \times 10^{3} \mathrm{CFU} / \mathrm{mL} ; 0.2 \% \text { Chlorhexidine: } \\
2.5 \times 10^{3} \mathrm{CFU} / \mathrm{mL} ; \text { Saline solution } 6.0 \times 10^{6} \mathrm{CFU} / \mathrm{mL}\end{array}$ \\
\hline
\end{tabular}


Table 8. Framework of studies. Distribution of promising EOs and their isolated constituents tested against caries-related bacteria.

\begin{tabular}{|c|c|c|c|c|c|c|c|c|c|c|c|}
\hline \multirow{3}{*}{$\begin{array}{c}\text { Plant Species or } \\
\text { Chemical Constituent }\end{array}$} & \multicolumn{11}{|c|}{ Antibacterial Efficacy } \\
\hline & \multicolumn{6}{|c|}{ Planktonic Cells } & \multicolumn{4}{|c|}{ Biofilms } & \multirow{2}{*}{ Clinical Trial } \\
\hline & Smu & Ssob & Ssan & Ssal & Lc & $\mathbf{L a}$ & Smu & Ssob & Ssal & Lc & \\
\hline A. ligustica & + & & & & & & & & & & \\
\hline B. dracunculifolia & + & & & & & & & & & & \\
\hline C. cajucara & + & + & & & + & & + & + & & + & \\
\hline C. japonica & + & + & + & & & & & & & & \\
\hline C. sativum & + & & & & & & & & & & \\
\hline E. caryophyllata & + & + & + & & & & & & & & \\
\hline L. sidoides & + & & & & & & & & & & Plaque reduction \\
\hline O. americanum & + & & & & + & & + & & & + & \\
\hline Menthol & + & & & & & + & & & & & \\
\hline Eugenol & + & + & + & & & & & & & & \\
\hline
\end{tabular}

Note: (+): MIC $<100 \mu \mathrm{g} / \mathrm{mL}$ or correspondent; Smu: S. mutans; Ssob: S. sobrinus; Ssan: S. sanguinis; Ssal: S. salivarius; Lc: L. casei; La: L. acidophilus.

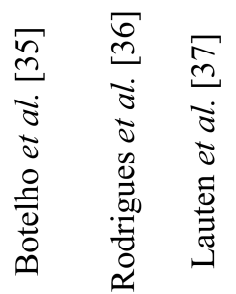

\begin{tabular}{|c|c|c|l|}
\hline+ & + & + & Random sequence generation (selection bias) \\
\hline$?$ & + & $?$ & Allocation concealment (selection bias) \\
\hline+ & + & + & Blinding of participants and personnel (performance bias) \\
\hline+ & + & + & Blinding of outcome assessment (detection bias) \\
\hline+ & + & + & Incomplete outcome data (attrition bias) \\
\hline+ & + & + & Selective reporting (reporting bias) \\
\hline 5 & 3 & 5 & Jadad Scale for assessment of quality \\
\hline
\end{tabular}

Figure 2. Risk-of-bias summary of the clinical trials included in this systematic review. Red (-) stands for high risk of bias, green $(+)$ stands for low risk of bias and yellow (?) stands for unclear risk of bias. Overall, the studies are compliant with the CONSORT guidelines for clinical trials of herbal interventions, showing low risk of bias. 
Table 9. Characteristics of the Randomized Clinical Trials included in this systematic review.

\begin{tabular}{|c|c|c|c|c|c|c|c|c|c|c|c|c|}
\hline Plant Species & $\begin{array}{l}\text { Essential Oil } \\
\text { Formulation }\end{array}$ & Study Design & Sample Size & Country & $\begin{array}{l}\text { Age (Mean } \pm \\
\text { SD)/Gender } \\
(\text { Fem }) *\end{array}$ & $\begin{array}{l}\text { Sample } \\
\text { Loss/Reasons }\end{array}$ & $\begin{array}{l}\text { Control } \\
\text { Group }\end{array}$ & Dosing Protocol & $\begin{array}{l}\text { Assessment } \\
\text { Checkpoints }\end{array}$ & $\begin{array}{l}\text { Assessment } \\
\text { Instruments of } \\
\text { Interest } \\
\end{array}$ & Outcome ** & Ref. \\
\hline Lipia sidoides & $\begin{array}{l}1 \% \text { L. sidoides } \\
\text { mouthrinse }\end{array}$ & $\begin{array}{l}\text { Phase II, } \\
\text { randomized, } \\
\text { double-blind, } \\
\text { crossover }\end{array}$ & $\begin{array}{l}n=55(n=27 \\
\text { L. sidoides } \\
\text { group; } n=28 \\
\text { control group) }\end{array}$ & Brazil & $\begin{array}{l}31 \pm 10.90 \\
55.6 \% \mathrm{~F}\end{array}$ & $\begin{array}{l}16 \text { individuals } \\
\text { (no gender } \\
\text { distinction)/lack of } \\
\text { compliance or } \\
\text { could not be } \\
\text { reached for } \\
\text { follow-up visits. }\end{array}$ & $0.12 \% \mathrm{CHX}$ & $\begin{array}{l}\text { Rinsing approx. } \\
15 \mathrm{~mL} \text { for } 30 \mathrm{~s} \text {, } \\
\text { twice a day (once } \\
\text { after breakfast and } \\
\text { once in the late } \\
\text { afternoon) during } \\
\text { a 7-day period. }\end{array}$ & $\begin{array}{l}\text { Baseline, } \\
1 \text { week }\end{array}$ & $\begin{array}{l}\text { Plaque index (PI) } \\
\text { measured at four } \\
\text { sites per tooth } \\
\text { (Ainamo \& Bay, } \\
1975 \text { ) }\end{array}$ & $+/+$ & [35] \\
\hline Lipia sidoides & $\begin{array}{l}10 \% \\
\text { L. sidoides gel }\end{array}$ & $\begin{array}{l}\text { Phase II, } \\
\text { randomized, } \\
\text { double-blind, } \\
\text { crossover. } \\
\text { Partial mouth } \\
\text { experimental } \\
\text { model }\end{array}$ & $\begin{array}{l}n=26(n=13 \\
\text { L. sidoides } \\
\text { group; } n=13 \\
\text { control group) }\end{array}$ & Brazil & $\begin{array}{l}22 \pm 4.24 \\
50.0 \% \mathrm{~F}\end{array}$ & $\begin{array}{l}4 \text { individuals } \\
\text { (no gender } \\
\text { distinction)/third } \\
\text { molar extraction }\end{array}$ & Placebo gel & $\begin{array}{l}\text { Filling a toothshield } \\
\text { with the gel prior to } \\
\text { insertion in the } \\
\text { mouth and } \\
\text { seating it over the } \\
\text { experimental teeth } 3 \\
\text { times a day for at } \\
\text { least } 1 \text { min. }\end{array}$ & $\begin{array}{l}\text { Baseline, } \\
3 \text { weeks }\end{array}$ & $\begin{array}{l}\text { Plaque index (PI) } \\
\text { measured at six } \\
\text { sites per tooth } \\
\text { (Turesky et al., } \\
1970)\end{array}$ & $-/+$ & {$[36]$} \\
\hline
\end{tabular}


Table 9. Cont.

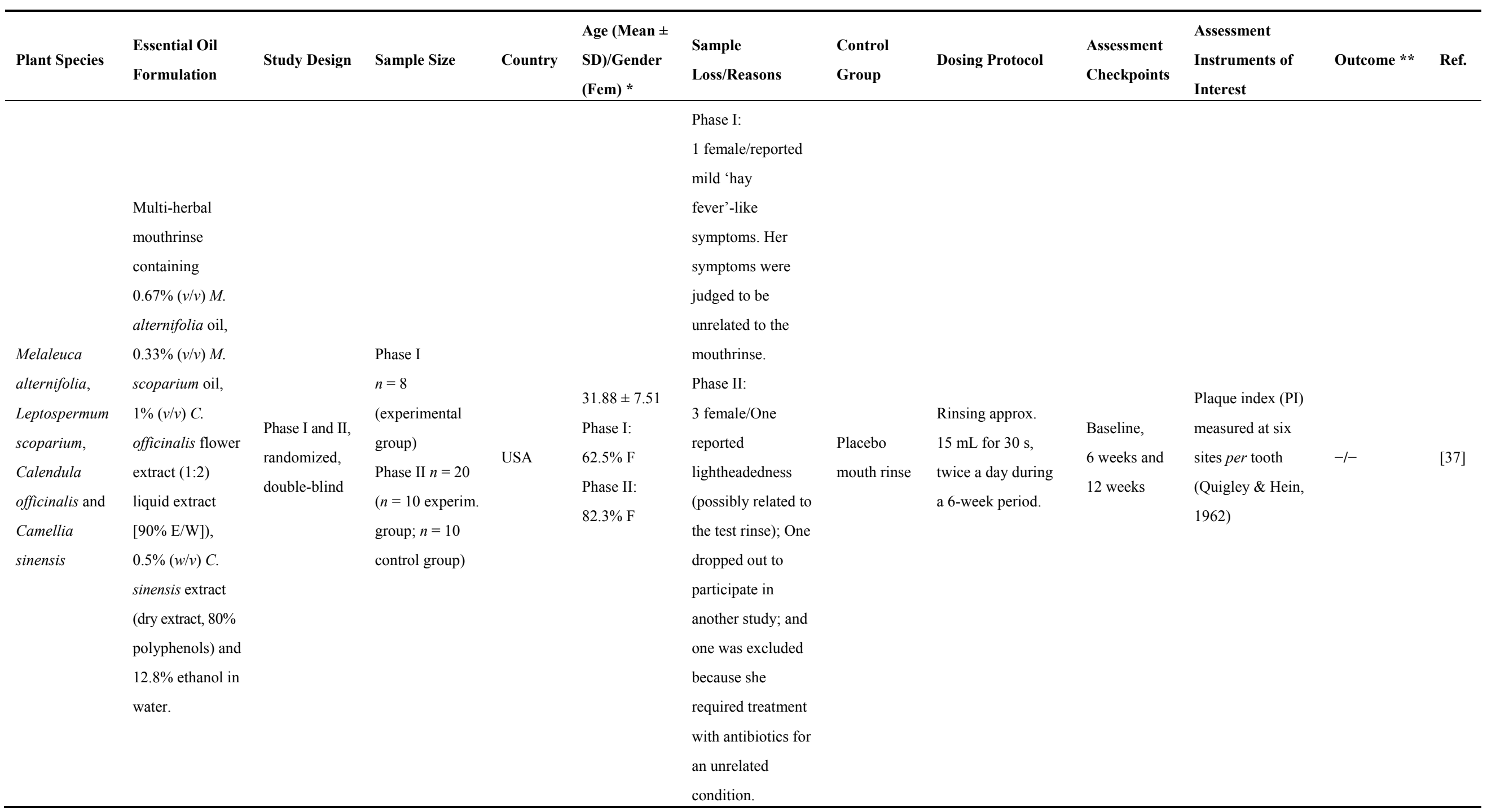

Note: CHX (chlorhexidine) mouthrinse; * Age and gender of individuals assigned to the experimental group; ** Statistically significant reduction $(+)$ or not $(-)$ in the amount of cariogenic biofilm compared to CHX or placebo (fist sign) and to the baseline condition (second sign, after slash) $(p<0.05$ ). 
The EO from A. camphorata, B. sulphurea, L. alba, M. glomerata, O. gratissimum and S. guianenses were not chemically characterized by the studies included in this review. Therefore, $21.7 \%$ of the selected studies had no chemical control regarding the EO under test. Furthermore, only $60.8 \%$ of the studies proceeded with a botanical identification of the aromatic plants that served as source for the EO. Finally, only $56.52 \%$ of the studies showed any piece of information about georeferencing of the plant species and $47.82 \%$ reported the period of plant collection.

\section{Discussion}

Essential oils have stood out as a promising source of bioactive molecules with potential application in the management of dental caries [40,41]. The data presented in this review suggest potential EO and constituents to be further tested as bioactive ingredients of anti-caries formulations. Moreover, the results of the reported chemical assessments of EO-isolated compounds could lead them to be used as chemical markers in future screening. Surprisingly, $20 \%$ and $60 \%$ of the studies do not provide any chemical or botanical information, respectively, which inevitably results in a biased and inconclusive analysis with reproducibility and traceability issues. Also, despite an understanding of the biological and physicochemical processes associated with the aetiopathogenesis of dental caries [8], great part (88\%) of the current evidence on the anti-caries potential of EO is based on in vitro studies rather than clinical trials (see Section 3.3 in this Discussion). Altogether, the benefits and issues related to EO research suggest wide avenues for scientists to work on more comprehensive and trustworthy bioprospection studies.

According to our searches, the majority of in vitro studies have evaluated the effect of EO or isolated compounds against $S$. mutans, as expected. Considered the most cariogenic of the oral streptococci, $S$. mutans colonizes the tooth surfaces and produces significant amounts of extra- and intra-cellular polysaccharides [42], being responsible for the initial stage of oral biofilm formation and carious lesions [43]. Nevertheless, other streptococci and lactobacilli species are also implicated on the onset [44] and progression [45] of caries, respectively, thus playing a role in the aetiopathogenesis of this biofilm-dependent disease. An EO of interest to be included in a formulation should be that able to affect bacterial virulence without suppressing the resident oral species, as a more specific therapeutic approach [8]. However, most studies provide just preliminary evidence of anti-caries activity without further assessing the effects of EO on putative virulence factors in cariogenic bacteria (e.g., glycosyltransferase and F-ATPase activity). In addition, the cariogenic biofilm is composed of a multi-species microbial community, in which the predominance of different microorganisms is changed as a function of host, diet and microorganism factors [46]. These aspects are not considered in most studies evaluating only planktonic cultures and, at most, monospecies biofilm cultures.

Next, we provide a brief summary of the plant species whose EO and their isolated compounds were found to have significant in vitro anti-caries potential. Attention is given to the ethnopharmacological knowledge, biological properties and chemical composition. Despite our attempts to make inter-study comparisons, there are underlying distinctions related to extraction methods, georeferencing, seasonality, which should be taken into account. 


\subsection{Promising Essential Oils against Cariogenic Bacteria}

Achillea ligustica (Asteraceae) is a small herbaceous plant rich in terpenes that grows in the Mediterranean region and has been used in folk medicine mainly for the treatment of gastrointestinal disorders [47]. The EO from different parts of this plant (inflorescences, leaves and flowers) is also found to have antimicrobial activity, particularly against $S$. mutans $[19,22]$. However, as it can be seen in this review, when the major compounds of A. ligustica EO are tested alone (e.g., $\gamma$-terpinene, $\beta$-pinene, 1,8-cineole, terpinen-4-ol), there is a decrease in their antimicrobial activity, which suggests a synergistic effect of the compounds present in the whole EO. Different EOs from the genus Achillea have been used in the cosmetic and liqueur industry as fragrances and flavoring agents, demonstrating commercial and economic relevance [22].

Baccharis dracunculifolia (Asteraceae) a native plant from Brazil, is widespread in the tropical areas of South America and is the botanical source of Southeastern (or green) propolis [48]. It has been widely used in folk medicine as febrifuge, anti-inflammatory, antiseptic and in the treatment of skin sores and gastrointestinal disorders [49]. The trans-nerolidol- and spathulenol-rich EO from B. dracunculifolia and its active fractions are bacteriostatic and have an in vitro anti-cariogenic activity by disrupting S. mutans biofilm at concentrations as low as $31.25 \mu \mathrm{g} / \mathrm{mL}$ [11].

Croton cajucara (Euphorbiaceae) is a common shrub growing in the Amazonian region commonly used in folk medicine as a tea for ailments such as diarrhea, diabetes and gastrointestinal disorders [50]. Alviano et al. [21] found that the EO of $C$. cajucara has significant antibacterial activity against S. mutans, S. sobrinus and L. casei in planktonic and monospecies biofilm cultures, unlike its isolated major compound linalool. This result disagrees with others reported in this review showing that linalool is considerably active against $S$. mutans [19,22,31]; however, it remains controversial.

Cryptomeria japonica (Cupressaceae) is an endemic and widely distributed coniferous plant in Japan, normally used for forestry, whose EO has been reported to have several pharmacological properties including larvicidal [51], antiulcer [52], antifungal [53] and antibacterial [20]. C. japonica EO is another example of how the complex mixture of chemical molecules plays a synergistic role in the antibacterial power of the EO over its isolated major compounds (sabinene, terpinen-4-ol, $\alpha$-pinene and $\alpha$-terpineol) [20]. In this review, we found significant inhibitory effects of the leaf EO against caries-related streptococci, warranting further investigation.

Coriandrum sativum (Apiaceae) popularly known as coriander, is an annual small plant whose leaves and seeds are widely used in folk medicine as anti-hypertensive, cholesterol-lowering and digestive stimulant [54], and also as food condiment. Moreover, other biological properties of C. sativum EO have also been reported: antifungal [55,56] antibacterial [11,56], antioxidant [57] and hepatoprotective [58]. The EO from $C$. sativum leaves contains mostly decanal, trans-2-decenal and 2-decen-1-ol [55], and has been shown to have in vitro anti-cariogenic potential against $S$. mutans biofilms and to be more active than its chemical fractions [11].

Eugenia caryophyllata (Myrtaceae) is widely cultivated in Indonesia, Sri Lanka, Madagascar, Tanzania and Brazil. E. caryophyllata EO (clove) has been described as having useful antiseptic, analgesic and anaesthetic effects. In community medicine, it serves as a topical pain-relieving and healing agent and in the industry as a fragrance and flavoring substance [59]. The main compounds of clove oil are phenylpropanoids such as eugenol and $\beta$-caryophyllene. According to our findings, 
eugenol was proven to be more active than the EO against S. mutans, i.e., showed lower MIC values. Nevertheless, the crude EO of E. caryophyllata, in general, showed strong antimicrobial activity against streptococci.

Lippia sidoides (Verbenaceae) is a typical shrub commonly found in the Northeastern Brazil, popularly used as topic skin and mucosal antiseptic [60]. L. sidoides EO also has anti-inflammatory, antioxidant and gastroprotective properties [61]. Its antimicrobial activity against cariogenic bacteria has been correlated with the presence of the phenolic monoterpenes thymol and carvacrol [62], and it may be considered of the most scientifically explored medicinal plants in Brazil, whose studies have reached the clinical phase. According to this review, L. sidoides EO showed both strong in vitro antibacterial activity and clinical efficacy as a mouthwash (see Section 3.3 in this Discussion), thus being considered a promising anti-plaque and anti-gingivitis phase II agent [37].

Ocimum americanum (Lamiaceae) popularly known as hoary basil, is an annual herbaceous plant native to Asia and Africa. O. americanum EO is reported to have anti-inflammatory, antinociceptive [63], antibacterial and insecticidal properties [64], and it is considered valuable for the cosmetic industry of soups and perfumes. The findings of this review showed that the leaf EO has strong antimicrobial activity against $S$. mutans and $L$. casei, either planktonic or biofilm cultures. The study by Thaweboon and Thaweboon [29] indicated that the 3\% leaf EO is as effective as $0.2 \%$ chlorhexidine in reducing the bacterial counting of cariogenic biofilm cultures of S. mutans and L. lactis, thus highlighting its potential as an antiseptic agent for oral care. Other studies in vitro and in vivo are now encouraged to elucidate its effects on other aspects related to the aetiopathogenesis of tooth decay (e.g., glucosyltransferase activity, acid production, enamel demineralization, among others).

Rosmarinus officinalis (Lamiaceae) is a culinary evergreen shrub native to the Mediterranean region that has also been used for medicinal purposes to treat bacterial and fungal infections [65]. Unlike the other cases presented thus far, the major compounds of $R$. officinalis EO (camphor, verbenone, $\alpha$-pinene, $\beta$-myrcene, 1,8 -cineole and $\beta$-caryophyllene) showed better activity (lower MIC value) against cariogenic bacteria—-particularly $S$. sobrinus and $S$. salivarius — than the crude EO.

\subsection{Promising Compounds Isolated from Essential Oils against Cariogenic Bacteria}

Generally, the major phytochemical compounds determine the biological properties of EOs [66]. In these cases, the study of isolated compounds is meaningful to concentrate the active principle, enable industrial scale production and allow improvements in the chemical structure using molecular engineering approaches. Here, we provide a summary on menthol and eugenol as the most outstanding compounds isolated from EOs that possess an anti-caries potential.

Menthol is a compound that has raised interest of the pharmaceutical and food industry in the last decades. It is a terpenoid that can be found in the EO of the Mentha spp. genus, such as peppermint, with a crystalline, clear or white-colored aspect (Figure 3). Although there are several isomers of menthol available, only (-)-menthol occurs in nature [34].

In vitro [34,67] and in situ [68] studies have demonstrated that menthol inhibits the growth of both Gram-positive and -negative bacteria and yeasts, and that its mechanism of action may be related to membrane disruption leading to cell leakage. A number of clinical trials [18] have also supported the use of this compound as an ingredient of mouthwash formulations; some of which are already 
commercially available worldwide. Although menthol has been used more as a flavoring agent than an active principle, it has been proven to have a considerable antimicrobial activity and is considered as GRAS (Generally Regarded as Safe) by the FDA (US Food and Drug Administration).

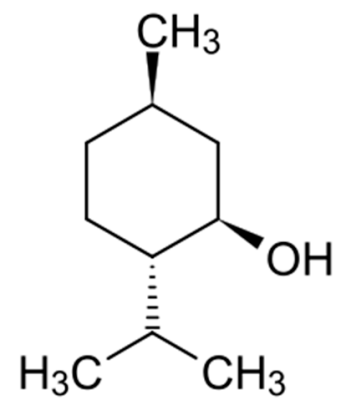

Figure 3. The chemical structure of (-)-menthol [(1R,2S,5R)-2-isopropyl-5-methylcyclohexanol].

Eugenol is an amphipathic phenolic compound (Figure 4) representing the major constituent of EO from clove (Eugenia caryophillis) and cinnamon (Cinnamomum zeylanicum) leaves [12]. Eugenol has been reported to have antiseptic, antimicrobial, anesthetic, analgesic, antioxidant, anti-inflammatory, and cardiovascular activities [69]. In dentistry, it is used as component of a cement containing zinc oxide for provisional sealing of cavities or as base for definitive fillings [70]. According to our review, eugenol has a promising antimicrobial activity against streptococci, particularly $S$. mutans, and should be considered as an anti-cariogenic agent to further clinical testing. It is an interesting source of new drugs as it is classified as GRAS by the FDA. This compound has been commercially marketed.

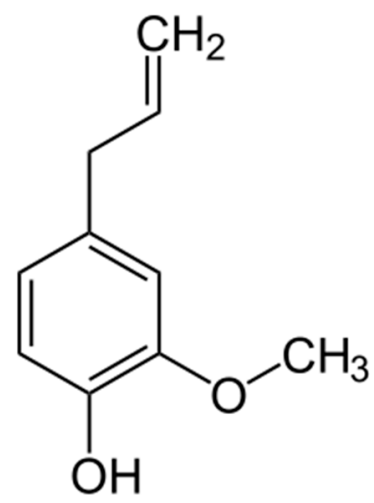

Figure 4. The chemical structure of eugenol [4-Allyl-2-methoxyphenol].

In addition to these three compounds, some others indicated in this review arouse attention for their antibacterial power with MIC values lower than $500 \mu \mathrm{g} / \mathrm{mL}$, as follows: 1,8, cineole, terpinen-4-ol, linalool, $\beta$-myrcene, $\beta$-caryophyllene and caryophyllene oxide. As such, the presence of these compounds in the $\mathrm{EO}$ of a plant could predict its antibacterial properties.

\subsection{Rational Clinical Use of Essential Oils and Isolated Compounds}

Despite the large number of in vitro studies on the antimicrobial activity of EOs, just a few reach the clinical phase and even fewer lead to a commercial product. Indeed, there is a small number of clinical trials reported in the literature aiming at the development of an EO-containing dental formulation. 
The most effective way to use the majority of EOs is by external application, such as mouthwashes for dental care. Topical application is generally safe [66] because most compounds are considered as GRAS by the FDA and have been long used in food preparation in several cultures. In case of eventual oral administration of a mouthwash, for instance, most EO compounds (such as (-)-menthol, thymol, carvacrol and eugenol) would be excreted renally or exhaled via the lungs [71,72], and their fast metabolism and short half-life highlight a minimal risk of accumulation in the organism [73]. However, although EOs have the advantage of being usually devoid of long-term cytotoxicity and genotoxic risks [12], the high volatility and chemical instability of some of their compounds in the presence of heat, humidity, light, or oxygen, may negatively impact their clinical use [74].

At the present time, the most popular EO-based formulation used in dental care in Western society is composed of a fixed combination of four EO-derived active ingredients: thymol (0.064\%), eucalyptol $(0.092 \%)$, methyl salicylate $(0.060 \%)$ and menthol $(0.042 \%)$. It is considered effective against cariogenic bacteria and relatively safe, although its $21 \%-27 \%$ alcoholic formula used to keep the constituents in solution is still controversial [75]. In some cases, such as with A. ligustica [19,22], C. japonica [20] and C. sativum [56], the synergism of compounds in the EO is critical for its biological properties as opposite to its isolated constituents. Such chemical complexity may favor solubility in vehicles other than ethanol (e.g., propylene glycol), with less likelihood of adverse effects.

According to our analysis, the mouthwash of thymol- and carvacrol-rich L. sidoides EO (ethanol-free) rinsed twice a day is an effective agent to prevent/disrupt the accumulation of cariogenic biofilm [36]. Furthermore, in a previous systematic review [76] we also found that such experimental mouthwash was effective against biofilm-induced gingivitis in adults. Altogether, these findings highlight the therapeutic potential of $L$. sidoides EO for dental care, but it is important to note that further studies are needed to investigate its effects on other aspects related to tooth decay, such as bacterial acid production, biofilm formation, enamel de- and remineralization, inhibition of glycosyltransferase production/activity, among others. Furthermore, the 10\% gel of thymol- and carvacrol-rich L. sidoides EO was not effective to reduce the amount of biofilm in adults compared to a placebo [37], suggesting that the pharmaceutical preparation plays a crucial role in this clinical outcome.

The synergistic association of EOs with other topical agents, e.g., fluoride, should also be considered for the management of dental caries, combining both antimicrobial and remineralization properties. A study by Zero et al. [77] showed that an EO mouthrinse with 100 parts per million fluoride should be effective in promoting enamel remineralization and fluoride uptake, thus providing anti-caries efficacy.

In dentistry, EOs could be useful as preoperative rinses, in periodontal procedures (e.g., sub-gingival irrigation), post-treatment applications, as a conventional mouthwash etc. Nevertheless, the majority of studies in the literature up to date fail to indicate robust and translational data to support the clinical use of novel EOs as ingredients of dental formulations, particularly against dental caries. With that said, this review suggests further research on the EOs and their constituents described earlier due to their favorable potential against streptococci and lactobacilli. In addition, it is important to determine the effects of EO on bacterial virulence factors related to dental caries, such as synthesis of extracellular polysaccharides and ability to survive in and produce acidic environments [8]. The scientific validation of the anti-caries activity of EOs and isolated compounds could provide not only patentable preparations and advances in preventive dentistry, but also commercial value. 


\section{Methods}

\subsection{Focused Question}

The aim of the present review was to answer the specific question: "Based on the current literature, which essential oils and/or isolated compounds are promising anti-caries agents warranting further investigation for clinical use?"

\subsection{Search Strategy and Selection of the Studies}

This systematic review of scientific studies followed the guidelines of the Transparent Reporting of Systematic Reviews and Meta-Analyses (PRISMA statement) [78]. Seven databases were systematically searched for clinical trials and in situ, in vivo and vitro studies (Table 10).

Table 10. Search strategy and bibliographic databases used to retrieve the articles falling into the scope of this systematic review.

\begin{tabular}{|c|c|}
\hline $\begin{array}{l}\text { Bibliographic Databases } \\
\text { (Primary Sources) }\end{array}$ & Search Strategy (Descriptors and Boolean Operators) \\
\hline $\begin{array}{l}\text { SciVerse Scopus (Since } 1995 \\
\text { until December 2014) }\end{array}$ & 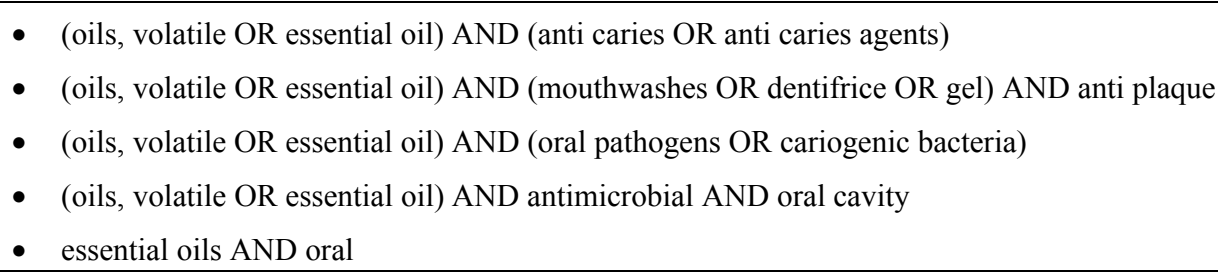 \\
\hline $\begin{array}{l}\text { Web of Science (Refine: } \\
\text { article or review) (Since } 1990 \\
\text { until December 2014) }\end{array}$ & $\begin{array}{l}\text { - (oils, volatile OR essential oil) AND (anti caries OR anti caries agents) } \\
\text { - (oils, volatile OR essential oil) AND (mouthwashes OR dentifrice OR gel) AND anti plaque } \\
\text { - (oils, volatile OR essential oil) AND (oral pathogens OR cariogenic bacteria) }\end{array}$ \\
\hline $\begin{array}{l}\text { Medline via Pubmed (Since } \\
1966 \text { until December 2014) }\end{array}$ & 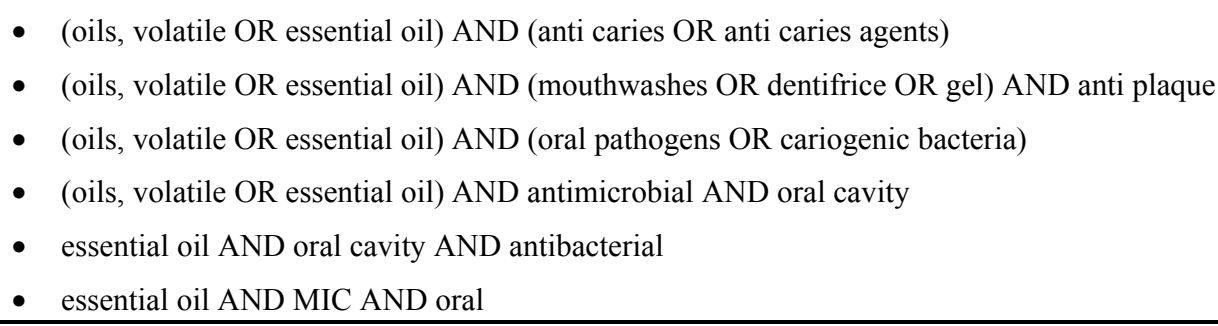 \\
\hline $\begin{array}{l}\text { SciELO (Scientific Electronic } \\
\text { Library Online) (Since } 1998 \\
\text { until December 2014) and } \\
\text { LILACS (Latin American and } \\
\text { Caribbean Health Sciences } \\
\text { Literature) (Since } 1982 \text { until } \\
\text { December 2014) }\end{array}$ & $\begin{array}{ll}\text { - } & \text { aceites esenciales } \\
\text { - } & \text { aceite volatile } \\
\text { - } & \text { essential oil AND caries } \\
\text { - } & \text { óleo essencial AND Streptococcus mutans } \\
\text { - } & \text { óleo essencial AND Lactobacillus } \\
\text { - } & \text { óleo essencial AND oral } \\
\text { - } & \text { óleo essencial AND antibacteriano } \\
\end{array}$ \\
\hline Cochrane Library & $\begin{array}{l}\text { - } \\
\text { - } \text { óleo essential oil AND caries } \\
\text { - óleo essencial AND Streptococcus mutans } \\
\text { - óleo essencial AND oral } \\
\text { - óleo essencial AND antibacteriano }\end{array}$ \\
\hline Google Scholar & - Manual searches according to the reference lists of the articles \\
\hline
\end{tabular}




\subsection{Eligibility Criteria}

A systematic selection of the articles was carried out by three independent examiners based on the following inclusion criteria: (1) Biological activity: anti-caries activity against oral microorganisms involved in the etiology and progression of dental caries; (2) Plant material and chemical assessment: essential oils and/or isolated compounds from aromatic plants (their chemical assessment was not a restricted inclusion criteria, instead, it served as a point for discussion); (3) Study design: In vitro, in situ and/or in vivo laboratorial studies (planktonic and biofilm assays); randomized controlled clinical trials (outcome of interest: reduction in the amount of cariogenic biofilm); (4) Methodological quality: For clinical trials, Jadad scale [79] equal to or greater than 3, meeting high quality standards (see Section 4.4 for details); accuracy of outcomes; internal and external validity; (5) Language: Articles written in English, Spanish or Portuguese; (6) Novelty: Novel essential oils-containing dental formulations were included, if not currently marketed. Examiners agreed that in cases of inconsistence the final verdict on which articles should be included in this review would be reached by consensus.

\subsection{Data Pooling and Analysis}

The data were allocated into worksheets to proceed with exploratory analysis according to the study design. For in vitro studies, in order to standardize the susceptibility patterns of microorganisms to essential oils or isolated compounds, we used their minimum inhibitory concentration (MIC) range as a parameter to determine the intensity of antibacterial activity, based on the literature [80] and on our research experience (Table 11). The retrieved data were expressed according to the bacterial species related to different types of tooth decay, in terms of selectivity to specific surfaces: Streptococcus mutans (sulcus and fissure, smooth surface caries - main etiological agent of dental caries) [81]; S. sanguinis, S. sobrinus, $S$. salivarius play a secondary role and may be recovered from sulcus, fissure and smooth surface caries [82]; Lactobacillus spp. (dentin and root surface caries) [45], either in planktonic or biofilm assays.

Table 11. Established parameters based on Minimum Inhibitory Concentrations of essential oils or related chemical constituents.

\begin{tabular}{ccc}
\hline MIC Range & Intensity of Antibacterial Activity & Score \\
\hline$\leq 100 \mu \mathrm{g} / \mathrm{mL}$ & very strong activity & $(++++)$ \\
$101-500 \mu \mathrm{g} / \mathrm{mL}$ & strong activity & $(+++)$ \\
$501-1000 \mu \mathrm{g} / \mathrm{mL}$ & moderate activity & $(++)$ \\
$1001-2000 \mu \mathrm{g} / \mathrm{mL}$ & weak activity & $(+)$ \\
$>2001 \mu \mathrm{g} / \mathrm{mL}$ & no activity & $(-)$ \\
\hline
\end{tabular}

For clinical trials, the data were analyzed based on the CONSORT guidelines for reporting randomized, controlled trials of herbal interventions [83]. Jadad Scale [79] has also been adopted in this review as it checks the validity of evidence on interventions and evaluates methodological quality (randomization, blinding and loss of follow-up). Based on these criteria, we assigned scores to the studies ranging from 0 to 5 . Studies reaching a score $<3$ were considered of poor quality and thus excluded from this review. Several studies, including systematic reviews, have already embraced 
this validated evaluation tool [84-87]. Furthermore, we used the risk-of-bias table proposed by Cochrane [88] to check the presence of selection, performance, detection, attrition and reporting biases in the selected clinical trials.

\section{Conclusions}

This review attempted to shed light on the anti-caries activity of EOs and their isolated constituents. Certainly, EOs extracted from a variety of aromatic plants worldwide can be considered promising sources of bioactive molecules effective against caries-related microorganisms, particularly S. mutans; however, most of the knowledge in the literature is based on in vitro studies and on a limited number of clinical trials. Overall, the studies have assessed the effects of EO and isolated compounds on microbial growth rather than virulence factors (e.g., bacterial EPS synthesis), which play a key role in the aetiopathogenesis of dental caries. Attention is also drawn to the fact that a number of studies do not provide any chemical or botanical characterization data, raising concern about the reproducibility and accuracy of their findings. Scientific journals should be more stringent in the adoption of criteria for the publication of studies with natural products, particularly EOs. Due to the gap between the in vitro biological properties identified in EOs and their clinical use for the prevention of dental caries, future researches should focus on translational approaches to advance the development of effective anti-caries products containing EO, given that most of them are considered as GRAS by the FDA.

\section{Acknowledgments}

Research reported in this publication was supported by São Paulo Research Foundation (FAPESP, Brazil, grants no. 2008/55492-7, no. 2011/14757-0, no. 2011/15984-0, no. 2013/25080-7); National Council for Scientific and Technological Development (CNPq, Brazil, grant no.308644/2011-5); and PROEX/CAPES 2242/2014 (grant no. 23038005263/2012-97).

\section{Author Contributions}

All authors contributed equally to this work.

\section{Conflicts of Interest}

The authors declare no conflict of interest.

\section{References}

1. National Institute of Health Consensus Development Panel. National Institutes of Health Consensus Development Conference statement. Diagnosis and management of dental caries throughout life. J. Am. Dent. Assoc. 2001, 132, 1153-1161.

2. Bagramian, R.A.; Garcia-Godoy, F.; Volpe, A.R. The global increase in dental caries. A pending public health crisis. Am. J. Dent. 2009, 22, 3-8.

3. Bönecker, M.; Tenuta, L.M.; Pucca Junior, G.A.; Costa, P.B.; Pitts, N. A social movement to reduce caries prevalence in the world. Braz. Oral Res. 2013, 27, 5-6.

4. Gunsolley, J.C. Clinical efficacy of antimicrobial mouthrinses. J. Dent. 2010, 38, S6-S10. 
5. Maguire, A. ADA clinical recommendations on topical fluoride for caries prevention. Evid. Based Dent. 2014, 15, 38-39.

6. Yévenes, I.; Alvarez, S.R.; Jara, M.N.; Wolfenson, P.M.; Smith, L.P. Comparison of mouthrinses containing chlorhexidine and other active agents with chlorhexidine mouthrinse-gel: Effects on de novo formation. J. Dent. Sci. 2009, 24, 345-348.

7. Samuels, N.; Grbic, J.T.; Saffer, A.J.; Wexler, I.D.; Williams, R.C. Effect of an herbal mouth rinse in preventing periodontal inflammation in an experimental gingivitis model: A pilot study. Compend. Contin. Educ. Dent. 2012, 33, 204-206, 208-211.

8. Jeon J.G.; Rosalen, P.L.; Falsetta, M.L.; Koo, H. Natural products in caries research: Current (limited) knowledge, challenges and future perspective. Caries Res. 2011, 45, 243-263.

9. Magee, P. Antiseptic drugs and disinfectants. Side Eff. Drugs Annu. 2007, 29, 241-243.

10. Bassolé, I.H.; Juliani, H.R. Essential oils in combination and their antimicrobial properties. Molecules 2012, 17, 3989-4006.

11. Galvão, L.C.C.; Furletti, V.F.; Bersan, S.M.F.; Cunha, M.G.; Ruiz, A.L.T.G.; Carvalho, J.E.; Sartoratto, A.; Rehder, V.L.G.; Figueira, G.M.; Duarte, M.C.T.; et al. Antimicrobial Activity of Essential Oils against Streptococcus mutans and their Antiproliferative Effects. Evid. Based Complement. Altern. Med. 2012, 12, 751435.

12. Bakkali, F.; Averbeck, S.; Averbeck, D.; Idaomar, M. Biological effects of essential oils-A review. Food Chem. Toxicol. 2008, 46, 446-475.

13. Sá, R.C.S.; Andrade, L.N.; Sousa, D.P. A review on anti-inflammatory activity of monoterpenes. Molecules 2013, 18, 1227-1254.

14. Pichersky, E.; Noel, J.P.; Dudareva, N. Biosynthesis of plant volatiles: Nature's diversity and ingenuity. Science 2006, 311, 808-811.

15. Bernardes, W.A.; Lucarini, R.; Tozatti, M.G.; Flauzino, L.G.; Souza, M.G.; Turatti, I.C.; Andrade e Silva, M.L.; Martins, C.H.; da Silva Filho, A.A.; Cunha, W.R. Antibacterial activity of the essential oil from Rosmarinus officinalis and its major components against oral pathogens. Z. Naturforschung C 2010, 65, 588-593.

16. Botelho, M.A.; Santos, R.A.; Martins, J.G.; Carvalho, C.O.; Paz, M.C.; Azenha, C.; Ruela, R.S.; Queiroz, D.B.; Ruela, W.S.; Marinho, G.; et al. Efficacy of a mouthrinse based on leaves of the neem tree (Azadirachta indica) in the treatment of patients with chronic gingivitis: A double-blind, randomized, controlled trial. J. Med. Plant Res. 2008, 2, 341-346.

17. Preus, H.R.; Koldsland, O.C.; Aass, A.M.; Sandvik, S.; Hansen, B.F. The plaque- and gingivitis-inhibiting capacity of a commercially available essential oil product. A parallel, split-mouth, single blind, randomized, placebo-controlled clinical study. Acta Odontol. Scand. 2013, 71, 1613-1619.

18. Van Leeuwen, M.P.; Slot, D.E.; van der Weijden, G.A. The effect of an essential-oils mouthrinse as compared to a vehicle solution on plaque and gingival inflammation: A systematic review and meta-analysis. Int. J. Dent. Hyg. 2014, 12, 160-167.

19. Cecchini, C.; Silvi, S.; Cresci, A.; Piciotti, A.; Caprioli, G.; Papa, F.; Sagratini, G.; Vittori, S.; Maggi, F. Antimicrobial efficacy of Achillea ligustica All. (Asteraceae) essential oils against reference and isolated oral microorganisms. Chem. Biodivers. 2012, 9, 12-24. 
20. Cha, J.D.; Jeong, M.R.; Jeong, S.I.; Moon, S.E.; Kil, B.S.; Yun, S.I.; Lee, K.Y.; Song, Y.H. Chemical composition and antimicrobial activity of the essential oil of Cryptomeria japonica. Phytother. Res. 2007, 21, 295-299.

21. Alviano, W.S.; Mendonça-Filho, R.R.; Alviano, D.S.; Bizzo, H.R.; Souto-Padrón, T.; Rodrigues, M.L.; Bolognese, A.M.; Alviano, C.S.; Souza, M.M. Antimicrobial activity of Croton cajucara Benth linalool-rich essential oil on artificial biofilms and planktonic microorganisms. Oral Microbiol. Immunol. 2005, 20, 101-105.

22. Maggi, F.; Bramucci, M.; Cecchini, C.; Coman, M.M.; Cresci, A.; Cristalli, G.; Lupidi, G.; Papa, F.; Quassinti, L.; Sagratini, G.; et al. Composition and biological activity of essential oil of Achillea ligustica All. (Asteraceae) naturalized in central Italy: Ideal candidate for anti-cariogenic formulations. Fitoterapia 2009, 80, 313-319.

23. Aguiar, G.P.; Carvalho, C.E.; Dias, H.J.; Reis, E.B.; Martins, M.H.; Wakabayashi, K.A.; Groppo, M.; Martins, C.H.; Cunha, W.R.; Crotti, A.E. Antimicrobial activity of selected essential oils against cariogenic bacteria. Nat. Prod. Res. 2013, 27, 1668-1672.

24. Shayegh, S.; Rasooli, I.; Taghizadeh, M.; Astaneh, S.D. Phytotherapeutic inhibition of supragingival dental plaque. Nat. Prod. Res. 2008, 22, 428-439.

25. Takarada, K.; Kimizuka, R.; Takahashi, N.; Honma, K.; Okuda, K.; Kato, T. A comparison of the antibacterial efficacies of essential oils against oral pathogens. Oral Microbiol. Immunol. 2004, 19, 61-64.

26. Moon, S.E.; Kim, H.Y.; Cha, J.D. Synergistic effect between clove oil and its major compounds and antibiotics against oral bacteria. Arch. Oral Biol. 2011, 56, 907-916.

27. Silvestri, J.D.F.; Paroul, N.; Czyewski, E.; Lerin, L.; Rotava, I.; Cansian, R.L.; Mossi, A.; Toniazzo, G.; Oliveira, D.; Treichel, H. Perfil da composição química e atividades antibacteriana e antioxidante do óleo essencial do cravo-da-índia (Eugenia caryophyllata Thunb.). Rev. Ceres 2010, 57, 589-594.

28. Hammer, K.A.; Dry, L.; Johnson, M.; Michalak, E.M.; Carson, C.F.; Riley, T.V. Susceptibility of oral bacteria to Melaleuca alternifolia (tea tree) oil in vitro. Oral Microbiol. Immunol. 2003, 18, 389-392.

29. Thaweboon, S.; Thaweboon, B. In vitro antimicrobial activity of Ocimum americanum L. essential oil against oral microorganisms. Southeast Asian J. Trop. Med. Public Health 2009, 40, 1025-1233.

30. Rasooli, I.; Shayegh, S.; Taghizadeh, M.; Astaneh, S.D.A. Rosemarinus officinalis and Thymus eriocalyx essential oils combat in vitro and in vivo dental biofilm formation. Pharmacogn. Mag. 2008, 14, 65-74.

31. Vagionas, K.; Graikou, K.; Ngassapa, O.; Runyoro, D.; Chinou, I. Composition and antimicrobial activity of the essential oils of three Satureja species growing in Tanzania. Food Chem. 2007, 103, 319-324.

32. Shapiro, S.; Meier, A.; Guggenheim, B. The antimicrobial activity of essential oils and essential oil components towards oral bacteria. Oral Microbiol. Immunol. 1994, 9, 202-208.

33. Roldán, L.P.; Díaz, G.J.; Duringer, J.M. Composition and antibacterial activity of essential oils obtained from plants of the Lamiaceae family against pathogenic and beneficial bacteria. Rev. Colomb. Cienc. Pecu. 2010, 23, 451-446. 
34. Al-Bayati, F.A. Isolation and identification of antimicrobial compound from Mentha longifolia L. leaves grown wild in Iraq. Ann. Clin. Microbiol. Antimicrob. 2009, 12, 8-20.

35. Botelho, M.A.; Bezerra-Filho, J.G.; Correa, L.L.; Fonseca, S.G.C.; Montenegro, D.; Gapski, R.; Brito, G.A.C.; Heukelbach, J. Effect of a novel essential oil mouthrinse without alcohol on gingivitis: A double-blinded randomized controlled trial. J. Appl. Oral Sci. 2007, 15, 175-180.

36. Rodrigues, I.S.C.; Tavares, V.N.; Pereira, S.L.S.; Costa, F.N. Antiplaque and antigingivitis effect of Lippia sidoides. A double-blind clinical study in humans. J. Appl. Oral Sci. 2009, 17, 404-407.

37. Lauten, J.D.; Boyd, L.; Hanson, M.B.; Lillie, D.; Gullion, C.; Madden, T.E. A Clinical Study: Melaleuca, Manuka, Calendula and Green Tea Mouth Rinse. Phytother. Res. 2005, 19, 951-957.

38. Lee, K.H.; Kim, B.S.; Keum, K.S.; Yu, H.H.; Kim, Y.H.; Chang, B.S.; Ra, J.Y.; Moon, H.D.; Seo, B.R.; Choi, N.Y.; et al. Essential oil of Curcuma longa inhibits Streptococcus mutans biofilm formation. J. Food Sci. 2011, 76, H226-H230.

39. Rasooli, I.; Shayegh, S.; Taghizadeh, M.; Astaneh, S.D. Phytotherapeutic prevention of dental biofilm formation. Phytother. Res. 2008, 22, 1162-1167.

40. Lang, G.; Buchbauer, G. A review on recent research results (2008-2010) on essential oils as antimicrobials and antifungals. A review. Flavour Fragr. J. 2012, 27, 13-39.

41. Lobo, P.L.; Fonteles, C.S.; Marques, L.A.; Jamacaru, F.V.; Fonseca, S.G.; de Carvalho, C.B.; de Moraes, M.E. The efficacy of three formulations of Lippia sidoides Cham. essential oil in the reduction of salivary Streptococcus mutans in children with caries: A randomized, double-blind, controlled study. Phytomedicine 2014, 21, 1043-1047.

42. Vu, B., Chen, M.; Crawford, R.J.; Ivanova, E.P. Bacterial extracellular polysaccharides involved in biofilm formation. Molecules 2009, 13, 2535-2554.

43. Loesche, W.J. Role of Streptococcus mutans in Human Dental Decay. Microbiol. Rev. 1986, 50, $353-380$.

44. Gross, E.L.; Beall, C.J.; Kutsch, S.R.; Firestone, N.D.; Leys, E.J.; Griffen, A.L. Beyond Streptococcus mutans: Dental Caries Onset Linked to Multiple Species by 16S rRNA Community Analysis. PLoS ONE 2012, 7, e47722.

45. Smith, S.I.; Aweh, A.J.; Coker, A.O.; Savage, K.O.; Abosede, D.A.; Oyedeji, K.S. Lactobacilli in human dental caries and saliva. Microbios 2001, 105, 77-85.

46. Marsh, P.D. Microbiology of Dental Plaque Biofilms and Their Role in Oral Health and Caries. Dent. Clin. North Am. 2010, 54, 441-454.

47. Lodi, G. Pianti Officinali Italiane, 2nd ed.; Edagricole: Bologna, Italy, 2001; p. 397.

48. Park, Y.K.; Alencar, S.M.; Aguiar, C.L. Botanical origin and chemical composition of Brazilian propolis. J. Agric. Food Chem. 2002, 50, 2502-2506.

49. Parreira, N.A.; Magalhães, L.G.; Morais, D.R.; Caixeta, S.C.; Sousa, J.P.; Bastos, J.K.; Cunha, W.R.; Silva, M.L.; Nanayakkara, N.P.; Rodrigues, V.; et al. Antiprotozoal, schistosomicidal, and antimicrobial activities of the essential oil from the leaves of Baccharis dracunculifolia. Chem. Biodivers. 2010, 7, 993-1001.

50. Di Stasi, L.C.; Santos, E.M.C.; Moreira dos Santos, C.; Hiruma, C.A. Plantas Medicinais da Amazônia; UNESP: São Paulo, Brazil, 1989; p. 127. 
51. Mdoe, F.P.; Cheng, S.S.; Lyaruu, L.; Nkwengulila, G.; Chang, S.T.; Kweka, E.J. Larvicidal efficacy of Cryptomeria japonica leaf essential oils against Anopheles gambiae. Parasites Vector. 2014, 4, 426, doi:10.1186/1756-3305-7-426.

52. Matsunaga, T.; Hasegawa, C.; Kawasuji, T.; Suzuki, H.; Saito, H.; Sagioka, T. Isolation of the antiulcer compound in essential oil from the leaves of Cryptomeria japonica. Biol. Pharm. Bull. 2000, 23, 595-598.

53. Cheng, S.S.; Lin, H.Y.; Chang, S.T. Chemical composition and antifungal activity of essential oils from different tissues of Japanese Cedar (Cryptomeria japonica). J. Agric. Food Chem. 2005, 53, 614-619.

54. Snigdha, C.; Monika, T. Coriandrum sativum: A promising functional and medicinal food. Int. J. Phytomed. Relat. Ind. 2013, 5, 59-65.

55. Freires, I.A.; Murata, R.M.; Furletti, V.F.; Sartoratto, A.; Alencar, S.M.; Figueira, G.M.; Rodrigues, J.A.O.; Duarte, M.C.T.; Rosalen, P.L. Coriandrum sativum L. (coriander) essential oil: Antifungal activity and mode of action on Candida spp., and molecular targets affected in human whole-genome expression. PLoS ONE 2014, 9, e99086, doi:10.1371/journal.pone.0099086.

56. Freires, I.A.; Bueno-Silva, B.; Galvão, L.C.C.; Duarte, M.C.T.; Sartoratto, A.; Figueira, G.M.; Alencar, S.M.; Rosalen, P.L. The effect of essential oils and bioactive fractions on Streptococcus mutans and Candida albicans biofilms: A confocal analysis. Evid. Based Complement. Altern. Med. 2015, 2015, 871316, doi:10.1155/2015/871316.

57. Harsha, S.N.; Anilakumar, K.R. In vitro free radical scavenging and DNA damage protective property of Coriandrum sativum L. leaves extract. J. Food Sci. Technol. 2012, 51, 1533-1539.

58. Sreelatha, S.; Padma, P.R.; Umadevi, M. Protective effects of Coriandrum sativum extracts on carbon tetrachloride-induced hepatotoxicity in rats. Food Chem. Toxicol. 2009, 47, 702-708.

59. Chaieb, K.; Hajlaoui, H.; Zmantar, T.; Kahla-Nakbi, A.B.; Rouabhia, M.; Mahdouani, K.; Bakhrouf, A. The chemical composition and biological activity of clove essential oil, Eugenia caryophyllata (Syzigium aromaticum L. Myrtaceae): A short review. Phytother. Res. 2007, 21, 501-506.

60. Lacoste, E.; Chaumont, J.P.; Mandin, D.; Plumel; M.M.; Matos, F.J.A. Antiseptic properties of essential oil of Lippia sidoides Cham. Application to the cutaneous microflora. Ann. Pharm. Fr. 1996, 54, 228-230.

61. Monteiro, M.V.; de Melo Leite, A.K.; Bertini, L.M.; de Morais, S.M.; Nunes Pinheiro, D.C. Topical anti-inflammatory, gastroprotective and antioxidant effects of the essential oil of Lippia sidoides Cham. leaves. J. Ethnopharmacol. 2006, 111, 378-382.

62. Botelho, M.A.; Nogueira, N.A.P.; Bastos, G.M.; Fonseca, S.G.C.; Lemos, T.L.G.; Matos, F.J.A.; Montenero, D.; Heukelback, J.; Rao, V.S.; Brito, G.A.C. Antimicrobial activity of the essential oil from Lippia sidoides, carvacrol and thymol against oral pathogens. Braz. J. Med. Biol. Res. 2007, 40, 349-356.

63. Yamada, A.N.; Grespan, R.; Yamada, A.T.; Silva, E.L.; Silva-Filho, S.E.; Damião, M.J.; de Oliveira Dalalio, M.M.; Bersani-Amado, C.A.; Cuman, R.K. Anti-inflammatory Activity of Ocimum americanum L. Essential Oil in Experimental Model of Zymosan-Induced Arthritis. Am. J. Chin. Med. 2013, 41, 913-926. 
64. Shadia, E.; El-Aziz, A.; Omer, E.A.; Sabra, A.S. Chemical Composition of Ocimum americanum Essential Oil and Its Biological Effects Against, Agrotis ipsilon, (Lepidoptera: Noctuidae). Res. J. Agric. Biol. Sci. 2007, 3, 740-747.

65. Bozin, B.; Mimmkica-Dukic, N.; Samojlik, I.; Jovin, E. Antimicrobial and antioxidant properties of rosemary and sage (Rosmarinus offcinalis L. and Salvia officinalis L., Lamiaceae) essential oils. J. Agric. Food Chem. 2007, 55, 7879-7885.

66. Bilia, A.R.; Guccione, C.; Isacchi, B.; Righeschi, C.; Firenzuoli, F.; Bergonzi, M.C. Essential oils loaded in nanosystems: A developing strategy for a successful therapeutic approach. Evid. Based Complement. Altern. Med. 2014, 2014, 651593, doi:10.1155/2014/651593.

67. Işcan, G.; Kirimer, N.; Kürkcüoğlu, M.; Başer, K.H.; Demirci, F. Antimicrobial screening of Mentha piperita essential oils. J. Agric. Food Chem. 2002, 50, 3943-3946.

68. Quintas, V.; Prada-López, I.; Prados-Frutos, J.C.; Tomás, I. In situ antimicrobial activity on oral biofilm: Essential oils vs. 0.2\% chlorhexidine. Clin. Oral Investig. 2015, 19, 97-107.

69. Pramod, K.; Ansari, S.H.; Ali, J. Eugenol: A natural compound with versatile pharmacological actions. Nat. Prod. Commun. 2010, 5, 1999-2006.

70. Souza-Costa, C.A.; Teixeira, H.M.; Nascimento, A.B.L.; Hebling, J. Biocompatibility of resin-based dental materials applied as liners in deep cavities prepared in human teeth. J. Biomed. Mater. Res. B 2007, 81, 175-184.

71. Kohlert, C.; Van Rensen, I.; März, R.; Schindler, G.; Graefe, E.U.; Veit, M. Bioavailability and pharmacokinetics of natural volatile terpenes in animals and humans. Planta Med. 2000, 66, 495-505.

72. Michiels, J.; Missotten, J.; Dierick, N.; Freumaut, D.; Maene, P.; Smet, S. In vitro degradation and in vivo passage kinetics of carvacrol, thymol, eugenol and trans-cinnamaldehyde along the gastrointestinal tract of piglets. J. Sci. Food Agric. 2008, 13, 2371-2381.

73. Kohlert, C.; Schindler, G.; März, R.W.; Abel, G.; Brinkhaus, B.; Derendorf, H.; Gräfe, E.U.; Veit, M. Systemic availability and pharmacokinetics of thymol in humans. J. Clin. Pharmacol. 2002, 42, 731-731.

74. Turek, C.; Stintzing, F.C. Stability of essential oils: A review. Compr. Rev. Food Sci. Food Saf. 2013, 12, 40-53.

75. Vlachojannis, C.; Winsauer, H.; Chrubasik, S. Effectiveness and safety of a mouthwash containing essential oil ingredients. Phytother. Res. 2013, 27, 685-691.

76. Freires, I.A.; Silva, I.C.; Alves, L.A.; Bezerra, L.M.; Castro, R.D. Clinical applicability of natural product(s)-containing mouthwashes as adjunctive treatment of biofilm-induced gingivitis: A systematic review. Braz. J. Med. Plants 2012, 14, 700-711.

77. Zero, D.T.; Zhang, J.Z.; Harper, D.S.; Wu, M.; Kelly, S.; Waskow, J.; Hoffman, M. The remineralizing effect of an essential oil fluoride mouthrinse in an intraoral caries test. $\mathrm{J}$. Am. Dent. Assoc. 2004, 135, 231-237.

78. Liberati, A.; Altman, D.G.; Tetzlaff, J.; Mulrow, C.; Gøtzsche, P.C.; Loannidis, J.P.A.; Clarke, M.; Devereaux, P.J.; Kleijnen, J.; Moher, D. The PRISMA statement for reporting systematic reviews and meta-analyses of studies that evaluate health care interventions: Explanation and elaboration. BMJ 2009, 339, b2700, doi:10.1136/bmj.b2700. 
79. Jadad, A.R.; Moore, R.A.; Carroll, D.; Jenkinson, C.; Reynolds, D.J.; Gavaghan, D.J.; McQuay, H.J. Assessing the quality of reports of randomized clinical trials: Is blinding necessary? Control. Clin. Trials 1996, 17, 1-12.

80. Duarte, M.C.T.; Leme, E.E.; Delarmelina, C.; Soares, A.A.; Figueira, G.M.; Sartoratto, A. Activity of essential oils from Brazilian medicinal plants on Escherichia coli. J. Ethnopharmacol. 2007, 111, 197-201.

81. Tanzer, J.M. Essential dependence of smooth surface caries on, and augmentation of fissure caries by, sucrose and Streptococcus mutans infection. Infect. Immun. 1979, 25, 526-531.

82. Thylstrup, A.; Fejerskov, O. Cariologia Clínica, 3rd ed.; Santos: São Paulo, Brazil, 2001; p. 421.

83. Gagnier, J.J.; Boon, H.; Rochon, P.; Moher, D.; Barnes, J.; Bombardier, C. Reporting Randomized, Controlled Trials of Herbal Interventions: An Elaborated CONSORT Statement. Ann. Intern. Med. 2006, 144, 364-367.

84. Linde, K.; Jonas, W.B.; Melchart, D.; Willich, S. The methodological quality of randomized controlled trials of homeopathy, herbal medicines and acupuncture. Int. J. Epidemiol. 2001, 30, 526-531.

85. Hu, J.K.; Chen, Z.X.; Zhou, Z.G.; Zhang, B.; Tian, J.; Chen, J.P.; Wang, L.; Wang, C.H.; Chen, H.Y.; Li, Y.P. Intravenous chemotherapy for resected gastric cancer: Meta-analysis of randomized controlled trials. World J. Gastroenterol. 2002, 8, 1023-1028.

86. Beek, D.V.; Gans, J.; McIntyre, P.; Prasad, K. Steroids in adults with acute bacterial meningitis: A systematic review. Lancet Infect. Dis. 2004, 4, 139-143.

87. Huntley, A.L.; Ernst, E. Soy for the treatment of perimenopausal symptoms-A systematic review. Maturitas 2004, 47, 1-9.

88. Higgins, J.P.T.; Green, S. Cochrane Handbook for Systematic Reviews of Interventions Version 5.0.1 [updated September 2008]. The Cochrane Collaboration, London, UK, 2008. Available online: Http://www.cochrane-handbook.org (accessed on 11 September 2014).

Sample Availability: Not available.

(C) 2015 by the authors; licensee MDPI, Basel, Switzerland. This article is an open access article distributed under the terms and conditions of the Creative Commons Attribution license (http://creativecommons.org/licenses/by/4.0/). 